\title{
AN ASYMPTOTIC INTEGRAL REPRESENTATION FOR CARLEMAN ORTHOGONAL POLYNOMIALS
}

\author{
ERWIN MIÑA-DÍAZ
}

\begin{abstract}
In this paper we investigate the asymptotic behavior of polynomials that are orthonormal over the interior domain of an analytic Jordan curve $L$ with respect to area measure. We prove that, inside $L$, these polynomials behave asymptotically like a sequence of certain integrals involving the canonical conformal map of the exterior of $L$ onto the exterior of the unit circle and certain meromorphic kernel function defined in terms of a conformal map of the interior of $L$ onto the unit disk. The error term in the integral representation is proven to decay geometrically and sufficiently fast, allowing us to obtain more precise asymptotic formulas for the polynomials under certain additional geometric considerations. These formulas yield, in turn, fine results on the location, limiting distribution and accumulation points of the zeros of the polynomials.
\end{abstract}

\section{INTRODUCTION}

Let $L_{1}$ be an analytic Jordan curve in the complex plane $\mathbb{C}$ and let $\Omega_{1}$ and $G_{1}$ be, respectively, the unbounded and bounded components of $\overline{\mathbb{C}} \backslash L_{1}$. Let $\left\{P_{n}(z)\right\}_{n=0}^{\infty}$ be the unique sequence of polynomials orthonormal over $G_{1}$, i.e., satisfying

$$
\begin{gathered}
P_{n}(z)=\kappa_{n} z^{n}+\text { lower degree terms, } \quad \kappa_{n}>0, \\
\frac{1}{\pi} \int_{G_{1}} P_{n}(z) \overline{P_{m}(z)} d A(z)= \begin{cases}0, & n \neq m, \\
1, & n=m,\end{cases}
\end{gathered}
$$

where $d A$ denotes the area measure.

These polynomials were first studied by Carleman [1] in 1922, who proved that they satisfy the strong asymptotic formula

$$
P_{n}(z)=\sqrt{n+1} \phi^{\prime}(z)[\phi(z)]^{n}[1+o(1)]
$$

locally uniformly as $n \rightarrow \infty$ on certain open set $\Omega_{\rho} \supset \bar{\Omega}_{1}$, where $\phi$ is the conformal map of $\Omega_{1}$ onto the exterior of the unit circle satisfying that $\phi(\infty)=\infty, \phi^{\prime}(\infty)>0$ (see Theorem 2.1 below for more details). In particular, it follows that the zeros of $P_{n}$ must accumulate as $n \rightarrow \infty$ on $\mathbb{C} \backslash \Omega_{\rho}$.

However, despite the fact that Carleman's work pioneered the study of polynomials orthogonal over planar domains, and that several subsequent works have been devoted to the subject (see, e.g., [6], [16], [18, [11, [8], [13]), no significant progress has been made in understanding the behavior of the polynomials $P_{n}(z)$ and its zeros in the orthogonality domain $G_{1}$ (more exactly, in $\mathbb{C} \backslash \Omega_{\rho}$ ). The aim of this paper is precisely to clarify this fundamental question.

The precise statements of our results are contained in Section 2 below. Roughly speaking, we show that the behavior of $P_{n}$ inside $G_{1}$ is governed by both the exterior map $\phi$ and certain meromorphic "kernel function" $L(\zeta, z)$, which is defined in terms of a conformal map $\varphi$ of $G_{1}$ onto the unit disk by

$$
L(\zeta, z):=\frac{\varphi^{\prime}(\zeta) \varphi^{\prime}(z)}{[\varphi(\zeta)-\varphi(z)]^{2}} .
$$

More exactly, $\phi$ and $L(\zeta, z)$ canalize their influence over $P_{n}$ through the asymptotic integral representation

$$
P_{n}(z)=\frac{(n+1)^{-1 / 2}}{2 \pi i} \oint_{L_{1}} L(\zeta, z)[\phi(\zeta)]^{n+1} d \zeta+o(1), \quad z \in G_{1} .
$$

The full version of (3), that is, with a good estimate on the rate of decay of the error term, is stated as Theorem 2.2 of Subsection 2.1.

Key words and phrases. Orthogonal polynomials; asymptotic behavior; integral representation; zeros of polynomials; equilibrium measure; Schwarz reflection principle; conformal map; lemniscate. 
We then exploit such a representation to obtain very detailed asymptotics of $P_{n}$ (see Theorems 2.4 and 2.5 of Subsection 2.1) valid for certain quite general sets $\Omega_{\rho}$ having piecewise analytic boundary $\left(\Omega_{\rho}\right.$ is the set on which Carleman's formula holds). Of particular interest is Theorem 2.4, which depicts the oscillatory behavior of $P_{n}$ in the interior of $\mathbb{C} \backslash \Omega_{\rho}$. As a consequence, fine results on the location, limiting distribution and accumulation points of the zeros of the polynomials follow. These are discussed in Subsection 2.2 .

In Subsection 2.3 we examine in detail the case of $L_{1}$ being the lemniscate $\left\{z:\left|z^{s}-1\right|=R^{s}\right\}$, where $s \geq 2$ is an integer and $R>1$. This example illustrates the situation in which Theorem 2.4 fails to describe the behavior of certain subsequences of $\left\{P_{n}\right\}_{n \geq 0}$. In particular, it exemplifies what could happen if a key hypothesis in the statement of some of the zero results fails to hold true. Finally, the proof of all the results are given in Section 3 .

It is important to remark that the results obtained in this paper have their counterparts for polynomials orthogonal over an analytic curve with respect to a positive analytic weight. These will appear in a paper to be submitted for publication soon.

We also remark that another important system of polynomials associated with a Jordan curve, the socalled Faber polynomials (see the monograph [19]), also satisfies an integral representation similar (though simpler and exact) to (3). In a recent paper [12], the author has exploited such a representation to derive precise asymptotic formulas for the Faber polynomials associated to a piecewise analytic curve. The results and techniques of proof in [12] exhibit a resemblance to those of the present paper that is worth noticing, since Faber polynomials have been often used as a tool for obtaining asymptotic properties of orthogonal polynomials over curves and regions.

\section{MAin RESUlts}

2.1. Asymptotic formulas. The following notation will be used throughout the paper. Given $r \geq 0$, we set

$$
\mathbb{T}_{r}:=\{w:|w|=r\}, \quad \mathbb{E}_{r}:=\{w: r<|w| \leq \infty\}, \quad \mathbb{D}_{r}:=\{w:|w|<r\} .
$$

If $K$ is a set and $f$ is a function defined on $K, \bar{K}$ denotes the closure of $K$ and $f(K):=\{f(z): z \in K\}$.

As in the introduction, $L_{1}$ is an analytic Jordan curve in the complex plane and $\Omega_{1}$ and $G_{1}$ are, respectively, the unbounded and bounded components of $\overline{\mathbb{C}} \backslash L_{1}$.

Let $\psi(w)$ be the unique conformal map of $\mathbb{E}_{1}$ onto $\Omega_{1}$ satisfying that $\psi(\infty)=\infty, \psi^{\prime}(\infty)>0$. Let $\rho \geq 0$ be the smallest number such that $\psi$ has an analytic and univalent continuation from $\mathbb{E}_{1}$ to $\mathbb{E}_{\rho}$. Because $L_{1}$ is analytic, $\rho<1$. For every $r \in[\rho, \infty)$, let

$$
\Omega_{r}:=\psi\left(\mathbb{E}_{r}\right), \quad L_{r}:=\partial \Omega_{r}, \quad G_{r}:=\mathbb{C} \backslash \bar{\Omega}_{r},
$$

and let $\phi(z): \Omega_{\rho} \mapsto \mathbb{E}_{\rho}$ be the inverse of $\psi$. Observe that, for every $r>1, L_{r}$ is an analytic Jordan curve.

The polynomials $P_{n}(z), n=0,1,2, \ldots$, that are orthonormal over $G_{1}$ with respect to area measure, that is, satisfying (11) and (2), were first considered by Carleman in [1]. We will refer to them as the Carleman polynomials 1 for the curve $L_{1}$ (or for the domain $G_{1}$ ).

Theorem 2.1. (Carleman [1]) The following asymptotic formulas hold true:

$$
\begin{gathered}
\kappa_{n}=\sqrt{n+1}\left[\phi^{\prime}(\infty)\right]^{n+1}\left[1+\mathcal{O}\left(\rho^{2 n}\right)\right], \\
P_{n}(z)=\sqrt{n+1} \phi^{\prime}(z)[\phi(z)]^{n}\left[1+h_{n}(z)\right], \quad z \in \Omega_{\rho},
\end{gathered}
$$

where $h_{n}(z)$ converges uniformly to zero as $n \rightarrow \infty$ on each $L_{r}, \rho<r<\infty$, with the following rate:

$$
h_{n}(z)= \begin{cases}\mathcal{O}\left(\rho^{n}\right), & r>1, \\ \mathcal{O}\left(\sqrt{n} \rho^{n}\right), & r=1, \\ \mathcal{O}\left(n^{-1 / 2}(\rho / r)^{n}\right), & \rho<r<1 .\end{cases}
$$

Note that, by the maximum modulus principle for analytic functions, the estimates given for $h_{n}(z)$ on $L_{r}$ are indeed valid on $\bar{\Omega}_{r}$. Carleman [1, Satz IV] stated and proved this theorem as holding uniformly in the exterior of the curve $L_{1}$ with the estimate $h_{n}(z)=\mathcal{O}\left(\sqrt{n} \rho^{n}\right)$. However, without any variation, his proof

\footnotetext{
${ }^{1}$ Carleman polynomials are also and often called Bergman polynomials.
} 
equally yields Theorem 2.1 in the way it has been stated above (see the first paragraph of Subsection 3.1 below preceding the proof of Theorem 2.2.).

So far as the author can learn, Carleman's formula is the only known result of substantial generality that neatly describes the asymptotic behavior of the polynomials $P_{n}$. Notice that, since both $\phi$ and $\phi^{\prime}$ do not vanish on $\Omega_{\rho}$, Theorem 2.1 implies that the zeros of $P_{n}$ must accumulate, in the limit, on $\mathbb{C} \backslash \Omega_{\rho}$. That is to say, any closed subset of $\Omega_{\rho}$ contains zeros of at most finitely many polynomials $P_{n}$. Our investigation focuses precisely in understanding how Carleman polynomials and their zeros behave in $\mathbb{C} \backslash \Omega_{\rho}$.

We now discuss a well-known important relation between Carleman polynomials and the conformal maps of $G_{1}$ onto the unit disk $\mathbb{D}_{1}$. Let $f$ be a function in the Bergman space $\mathcal{B}^{2}\left(G_{1}\right)$ of the domain $G_{1}$, that is, $f$ is and analytic function defined on $G_{1}$ satisfying

$$
\frac{1}{\pi} \int_{G_{1}}|f(\zeta)|^{2} d A(\zeta)<\infty
$$

and let $\tau=\tau(f) \geq 1$ be the largest number such that $f$ has an analytic continuation to $G_{\tau}$. It is well-known [21, pp. 128-131] that

$$
f(\zeta)=\sum_{k=0}^{\infty} \alpha_{k}(f) P_{k}(\zeta), \quad \zeta \in G_{\tau}
$$

where

$$
\alpha_{k}(f)=\frac{1}{\pi} \int_{G_{1}} f(\zeta) \overline{P_{k}(\zeta)} d A(\zeta), \quad k=0,1,2, \ldots
$$

and the series in the right-hand side of (6) converges locally uniformly to $f$ on $G_{\tau}$.

Let us then apply this result to the so-called Bergman kernel function $K(\zeta, z)$ of the space $\mathcal{B}^{2}\left(G_{1}\right)$ (see [4. pp. 30-33]), which has the reproducing property

$$
f(z)=\frac{1}{\pi} \int_{G_{1}} f(\zeta) \overline{K(\zeta, z)} d A(\zeta) \quad \forall f \in \mathcal{B}^{2}\left(G_{1}\right), z \in G_{1},
$$

and can be expressed in terms of a conformal map $\varphi$ of $G_{1}$ onto $\mathbb{D}_{1}$ as

$$
K(\zeta, z)=\frac{\overline{\varphi^{\prime}(z)} \varphi^{\prime}(\zeta)}{[1-\overline{\varphi(z)} \varphi(\zeta)]^{2}}, \quad \zeta, z \in G_{1}
$$

Because $L_{1}$ is a Jordan curve, any such map $\varphi$ can be extended as a continuous and bijective function $\varphi: \bar{G}_{1} \rightarrow \overline{\mathbb{D}}_{1}$. Moreover, being $L_{1}$ analytic, $\varphi$ has a meromorphic continuation to $G_{1 / \rho}$, which is indeed given by

$$
\varphi(z)=\frac{1}{\overline{\varphi\left(z^{*}\right)}}, \quad z \in G_{1 / \rho} \backslash \bar{G}_{1}
$$

where

$$
z^{*}=\psi(1 / \overline{\phi(z)}), \quad z \in G_{1 / \rho} \cap \Omega_{\rho},
$$

is the Schwarz reflection about $L_{1}$ of any point $z \in G_{1 / \rho} \cap \Omega_{\rho}$ (see [3] for details).

It follows from these considerations and relation (8) that for every $z \in G_{1}, K(\cdot, z)$ is analytic on $\bar{G}_{1}$, and since by (7), $\alpha_{k}(K(\cdot, z))=\overline{P_{k}(z)}$, we obtain from (6) that

$$
\frac{\overline{\varphi^{\prime}(z)} \varphi^{\prime}(\zeta)}{[1-\overline{\varphi(z)} \varphi(\zeta)]^{2}}=\sum_{k=0}^{\infty} \overline{P_{k}(z)} P_{k}(\zeta), \quad \zeta \in \bar{G}_{1}, \quad z \in G_{1}
$$

with the series converging uniformly in $\zeta \in \bar{G}_{1}$ for each fixed $z \in G_{1}$.

As we shall see soon, it turns out that the behavior of $P_{n}$ in $G_{1}$ is closely related to a symmetric "meromorphic kernel" $L(\zeta, z)$ that well resembles the Bergman kernel, namely, the function of two variables

$$
L(\zeta, z):=\frac{\varphi^{\prime}(\zeta) \varphi^{\prime}(z)}{[\varphi(\zeta)-\varphi(z)]^{2}}, \quad \zeta, z \in G_{1}
$$


That $L(\zeta, z)$ does not depend on the choice of the conformal map $\varphi$ can be easily established from the fact that any two conformal maps $\varphi$ and $\varphi_{1}$ of $G_{1}$ onto $\mathbb{D}_{1}$ are related through a Möbius transformation, that is,

$$
\varphi(z)=e^{i \theta} \frac{\varphi_{1}(z)-\varphi_{1}\left(z_{0}\right)}{1-\overline{\varphi_{1}\left(z_{0}\right)} \varphi_{1}(z)}, \quad e^{i \theta}=\varphi^{\prime}\left(z_{0}\right)\left(1-\left|\varphi_{1}\left(z_{0}\right)\right|^{2}\right) / \varphi_{1}^{\prime}\left(z_{0}\right)
$$

where $z_{0}$ is that point of $G_{1}$ mapped by $\varphi$ onto 0 .

If we specifically choose a map $\varphi$ that does not vanish on $G_{1} \cap \Omega_{\rho}$, then in view of (9) and (10), $\varphi$ has a one-to-one analytic continuation to $G_{1 / \rho}$, so that $L(\zeta, z)$ can be extended as a continuous function

$$
L(\zeta, z): G_{1 / \rho} \times G_{1 / \rho} \rightarrow \overline{\mathbb{C}}
$$

such that for every fixed $z \in G_{1 / \rho}, L(\cdot, z)$ is analytic on $G_{1 / \rho} \backslash\{z\}$ with a Laurent expansion at $z$ of the form

$$
L(\zeta, z)=\frac{1}{(\zeta-z)^{2}}+a_{0}+a_{1}(\zeta-z)+a_{2}(\zeta-z)^{2}+\cdots .
$$

We combine Theorem 2.1 with relation (11) to deduce the following theorem:

Theorem 2.2. With the notations above, we have that

$$
P_{n}(z)=\frac{(n+1)^{-1 / 2}}{2 \pi i} \oint_{L_{1}} L(\zeta, z)[\phi(\zeta)]^{n+1} d \zeta+\epsilon_{n}(z), \quad z \in G_{1}
$$

where the functions $\epsilon_{n}(z), n=0,1,2, \ldots$, are analytic on $G_{1 / \rho}$ and have the following property: if $E \subset G_{1 / \rho}$ is such that for some $0 \leq \tau<1 / \rho$,

$$
P_{n}(z)=\mathcal{O}\left(\sqrt{n} \tau^{n}\right)
$$

uniformly on $E$ as $n \rightarrow \infty$, then

$$
\epsilon_{n}(z)=\mathcal{O}\left(\sqrt{n}(\tau \rho)^{n}\right)
$$

uniformly on $E$ as $n \rightarrow \infty$.

A simple consequence of Theorem 2.2 is the following improvement in Carleman's formula regarding the error estimate:

Corollary 2.3. Indeed, for the functions $h_{n}(z)$ defined by (4), we have that $h_{n}(z)=\mathcal{O}\left(\rho^{n}\right)$ uniformly in $z \in L_{1}$ as $n \rightarrow \infty$.

From Theorem 2.1 and the maximum modulus principle for analytic functions we see that if $\rho<r<1$, then $P_{n}(z)=\mathcal{O}\left(\sqrt{n} r^{n}\right)$ uniformly in $z \in \bar{G}_{r}$ as $n \rightarrow \infty$. Consequently, it follows from Theorem 2.2 after integrating by parts over $L_{1}$ and making the change of variables $\zeta=\psi(t)$ that

$$
\begin{aligned}
P_{n}(z) & =\frac{\sqrt{n+1} \varphi^{\prime}(z)}{2 \pi i} \oint_{L_{1}} \frac{\phi^{\prime}(\zeta)[\phi(\zeta)]^{n} d \zeta}{\varphi(\zeta)-\varphi(z)}+\epsilon_{n}(z) \\
& =\frac{\sqrt{n+1} \varphi^{\prime}(z)}{2 \pi i} \oint_{\mathbb{T}_{1}} \frac{t^{n} d t}{\varphi(\psi(t))-\varphi(z)}+\mathcal{O}\left(\sqrt{n}(r \rho)^{n}\right), \quad z \in \bar{G}_{r}, \quad \rho<r<1 .
\end{aligned}
$$

To illustrate how effectively Theorem 2.2 can be exploited for deriving finer asymptotic results, let us consider the situation in which the boundary $L_{\rho}$ of $\Omega_{\rho}$ is a piecewise analytic curve without cusps or smooth corners (see, however, Remark 2.6 below). More precisely, we shall assume $\psi$ satisfies Conditions A.1 and A.2 to be stated in what follows.

We define an analytic arc as being the image of the interval $[0,1]$ by a function $f(t)$ analytic in $[0,1]$ such that $f^{\prime}(t) \neq 0$ for all $t \in[0,1]$ and $f\left(t_{1}\right) \neq f\left(t_{2}\right)$ for all $0<t_{1}<t_{2}<1$. The endpoints of the arc are $f(0)$ and $f(1)$, which may coincide. We call the arc simple if $f$ is one-to-one on $[0,1]$. Notice that, according to this definition, an analytic Jordan curve is also an analytic arc. Our first assumption is:

A.1: The map $\psi$ has a continuous extension to $\overline{\mathbb{E}}_{\rho}$ and there are $s \geq 1$ distinct points $\omega_{1}, \omega_{2}, \ldots, \omega_{s}$ in $\mathbb{T}_{\rho}$ such that if $w_{1} \neq w_{2}$ are two points of $\mathbb{T}_{\rho}$ for which $\psi\left(w_{1}\right)=\psi\left(w_{2}\right)$, then $\left\{w_{1}, w_{2}\right\} \subset\left\{\omega_{1}, \ldots, \omega_{s}\right\}$. Moreover, if $\ell$ is any of the $s$ open circular arcs that compose $\mathbb{T}_{\rho} \backslash\left\{\omega_{1}, \omega_{2}, \ldots, \omega_{s}\right\}$, say with endpoints $\omega_{k}, \omega_{j}$, then $\psi(\bar{\ell})$ is an analytic arc with endpoints $\psi\left(\omega_{k}\right), \psi\left(\omega_{j}\right)$ (see Figure 1 below). 


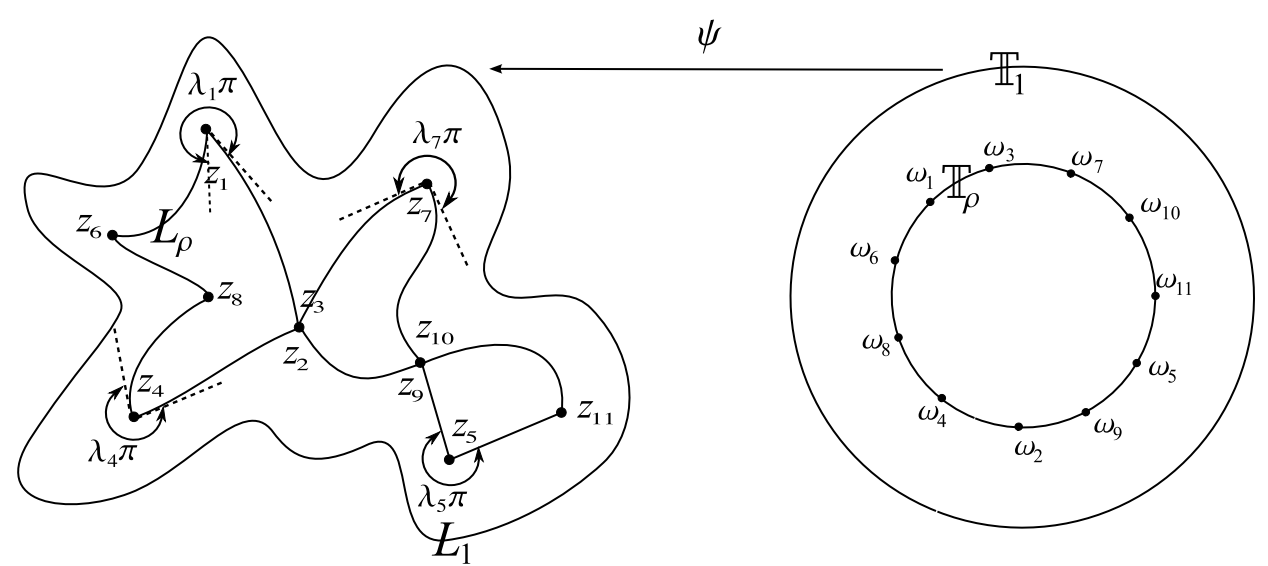

Figure 1. Illustration of a map $\psi$ satisfying Conditions A.1 and A.2.

Thus, $L_{\rho}=\partial \Omega_{\rho}$ is a piecewise analytic curve. Let $z \in L_{\rho}$ and $w=\rho e^{i \Theta}$ be such that $z=\psi(w)$. The exterior angle at $z$ relative to $w$ is defined to be that angle $\alpha \in[0,2 \pi]$ such that

$$
\arg \left[\psi\left(\rho e^{i \theta}\right)-z\right] \rightarrow \begin{cases}\beta & \text { as } \theta \rightarrow \Theta- \\ \beta+\alpha & \text { as } \theta \rightarrow \Theta+\end{cases}
$$

Let

$$
z_{k}:=\psi\left(\omega_{k}\right), \quad k \in\{1,2, \ldots, s\},
$$

be "the corners of $L_{\rho}$ ". Notice that they are not necessarily pairwise distinct.

For each $k \in\{1,2, \ldots, s\}$, let $\lambda_{k} \in[0,2]$ be such that $\lambda_{k} \pi$ is the exterior angle at $z_{k}$ relative to $\omega_{k}$. Our second assumption on $\psi$ is:

A.2: $\lambda_{k} \notin\{0,1,2\}$ for every $k \in\{1,2, \ldots, s\}$ (i.e., $L_{\rho}$ has no cusps or smooth corners).

We assume that the $\omega_{k}$ 's have been indexed in such a way that for some $u \in\{1,2, \ldots, s\}$,

$$
\lambda_{1}=\lambda_{2}=\cdots=\lambda_{u}<\lambda_{u+1} \leq \cdots \leq \lambda_{s} .
$$

Let $\Theta_{1}, \Theta_{2}, \ldots, \Theta_{s}$ be the arguments of the numbers $\omega_{k}$, that is,

$$
\omega_{k}=\rho e^{i \Theta_{k}}, \quad 0 \leq \Theta_{k}<2 \pi, \quad 1 \leq k \leq s .
$$

By a well-known result of Lehman (see [7] and Subsection 3.2 below), the map $\psi(w)$ admits an asymptotic expansion about each $\omega_{k}$. In particular, for each $k \in\{1,2, \ldots, s\}$, the limit

$$
A_{k}:=\lim _{\substack{w \rightarrow \omega_{k} \\ w \in \mathbb{E}_{\rho}}} \frac{\psi(w)-z_{k}}{\left(w-\omega_{k}\right)^{\lambda_{k}}}
$$

is a complex number different from zero. Obviously, the value of this number $A_{k}$ depends on the branch chosen for the function $\left(w-\omega_{k}\right)^{\lambda_{k}}$ in a $\delta$-neighborhood of the form $\left\{w \in \mathbb{E}_{\rho}: 0<\left|w-\omega_{k}\right|<\delta\right\}$. Here we choose the one corresponding to the branch of the argument

$$
\Theta_{k}-\pi<\arg \left(w-\omega_{k}\right)<\Theta_{k}+\pi, \quad w \in \mathbb{C} \backslash\left\{t \omega_{k}: t \leq 1\right\} .
$$

The symbol $\left(\begin{array}{l}a \\ b\end{array}\right)$ stands for the generalized binomial coefficient, i.e.,

$$
\left(\begin{array}{l}
a \\
b
\end{array}\right):=\frac{\Gamma(a+1)}{\Gamma(b+1) \Gamma(a-b+1)},
$$

where $\Gamma$ denotes the Euler gamma function.

The behavior of $P_{n}$ inside $G_{\rho}$ is given first. It strongly reflects the dependance of $P_{n}$ on both $\phi$ and $L(\zeta, z)$.

Theorem 2.4. For every $z \in G_{\rho}$, we have

$$
\frac{P_{n}(z)}{\sqrt{n+1}\left(\begin{array}{c}
n \\
-\lambda_{1}-1
\end{array}\right) \rho^{n+1+\lambda_{1}}}=-\sum_{k=1}^{u} L\left(z_{k}, z\right) A_{k} e^{i\left(n+1+\lambda_{1}\right) \Theta_{k}}+R_{n}(z),
$$


where

$$
R_{n}(z)=\left\{\begin{array}{ll}
\mathcal{O}\left(n^{-\lambda_{1}}\right), & \text { if } 0<\lambda_{1}<1, \lambda_{1} \neq 1 / 2, \\
\mathcal{O}\left(n^{-1} \log n\right), & \text { if } \lambda_{1}=1 / 2, \\
\mathcal{O}\left(n^{-1}\right), & \text { if } 1<\lambda_{1}<2,
\end{array}+ \begin{cases}\mathcal{O}\left(n^{\lambda_{1}-\lambda_{u+1}}\right), & \text { if } u<s \\
0, & \text { if } u=s\end{cases}\right.
$$

uniformly as $n \rightarrow \infty$ on compact subsets of $G_{\rho}$.

To state the behavior of $P_{n}(z)$ for points $z$ near $L_{\rho}$ we need the following piece of notation. For $\delta \in(0, \rho)$ and $1 \leq k \leq s$, we define the following open "pie-slices":

$$
\Sigma_{\delta, k}:=\left\{w: \rho-\delta<|w|<\rho^{2} /(\rho-\delta), \Theta_{k}-\delta<\arg (w)<\Theta_{k}+\delta\right\} .
$$

Theorem 2.5. (a) For every $\delta \in(0, \rho)$, there exists $\sigma \in(\rho-\delta, \rho)$ such that $\psi$ has a one-to-one analytic continuation to $\mathbb{E}_{\sigma} \backslash \cup_{k=1}^{s} \overline{\Sigma_{\delta, k}}$, and if

$$
V_{\delta, \sigma}:=\left\{z=\psi(w): w \in \mathbb{E}_{\sigma} \backslash \cup_{k=1}^{s} \overline{\Sigma_{\delta, k}}\right\}
$$

and $\phi$ denotes the inverse of $\psi$, then

$$
\frac{P_{n}(z)}{\sqrt{n+1}}=\phi^{\prime}(z)[\phi(z)]^{n}-\left(\begin{array}{c}
n \\
-\lambda_{1}-1
\end{array}\right) \rho^{n+1+\lambda_{1}}\left(\sum_{k=1}^{u} L\left(z_{k}, z\right) A_{k} e^{i\left(n+1+\lambda_{1}\right) \Theta_{k}}+R_{n}(z)\right), \quad z \in V_{\delta, \sigma} \cap G_{1},
$$

where $R_{n}(z)$ satisfies (16) locally uniformly on $V_{\delta, \sigma} \cap G_{1}$ as $n \rightarrow \infty$.

(b) For every corner $z_{j}$,

$$
\frac{P_{n}\left(z_{j}\right)}{\sqrt{n+1}\left(\begin{array}{c}
n \\
\lambda_{j}^{*}-1
\end{array}\right) \rho^{n+1-\lambda_{j}^{*}}}=\sum_{\substack{k: z_{k}=z_{j} \\
\lambda_{k}=\lambda_{j}^{*}}}\left(A_{k}\right)^{-1} e^{i\left(n+1-\lambda_{j}^{*}\right) \Theta_{k}}+o(1)
$$

where $\lambda_{j}^{*}=\max \left\{\lambda_{k}: z_{k}=z_{j}, 1 \leq k \leq s\right\}$.

The rate of decay of the $o(1)$-error term in (18) can be estimated by comparing the terms in the right-hand side of (71).

Remark 2.6. Totally similar results can be obtained for considerably more general piecewise analytic curves $L_{\rho}$, including those having inner cusps (as viewed from $\Omega_{\rho}$ ) and smooth corners, more specifically, under the following assumptions:

A.1': The map $\psi$ has a continuous extension to $\overline{\mathbb{E}}_{\rho}$ and there are $s \geq 1$ distinct points $\omega_{1}, \omega_{2}, \ldots, \omega_{s}$ in $\mathbb{T}_{\rho}$ such that if $\ell$ is any of the $s$ open circular arcs that compose $\mathbb{T}_{\rho} \backslash\left\{\omega_{1}, \omega_{2}, \ldots, \omega_{s}\right\}$, say with endpoints $\omega_{k}, \omega_{j}$, then $\psi$ is one-to-one on $\ell$ and $\psi(\bar{\ell})$ is an analytic arc with endpoints $\psi\left(\omega_{k}\right), \psi\left(\omega_{j}\right)$.

A.2': $\lambda_{k}>0$ for every $k \in\{1,2, \ldots, s\}$, and if $\lambda_{k} \in\{1,2\}$ for all $k \in\{1,2, \ldots, s\}$, then there is at least one $k$ for which logarithmic terms occur in the Lehman expansion of $\psi$ about $\omega_{k}$.

The statements of the corresponding results and their proofs are, however, more cumbersome, and so we have sacrificed generality in the present paper for the benefit of clarity. The interested reader will find useful to consult [12, where similar asymptotic formulas have been derived for the Faber polynomials of a domain $\Omega_{\rho}$ satisfying A.1' and A.2' by exploiting an integral representation for these polynomials that is somewhat similar to (13).

2.2. The zeros of $P_{n}(z)$. Throughout this subsection, we assume that the map $\psi$ satisfies Conditions A.1 and A.2 stated in Subsection 2.1. Here we shall discuss some of the conclusions that can be drawn from our previous results regarding the location, limiting distribution and accumulation points of the zeros of Carleman polynomials.

Asymptotic formulas similar to (15) and (17) are known to be satisfied by other important systems of polynomials, e.g., polynomials orthogonal on the unit circle with respect to certain types of weights [20], 9, [10], and Faber polynomials for domains with piecewise analytic boundary [12. The results that follow are well-known consequences of such type of behavior.

We start by introducing the notation and concepts needed in analyzing the zeros of $P_{n}$. The letter $\mathcal{Z}$ denotes the set of accumulation points of the zeros of Carleman polynomials, i.e., $\mathcal{Z}$ consists of all points $t \in \overline{\mathbb{C}}$ such that every neighborhood of $t$ contains zeros of infinitely many polynomials $P_{n}$. 
We shall denote by $\nu_{n}$ the normalized counting measure of the zeros of $P_{n}$, that is,

$$
\nu_{n}:=\frac{1}{n} \sum_{k=1}^{n} \delta_{z_{k, n}}, \quad n=1,2, \ldots
$$

where $z_{1, n}, z_{2, n}, \ldots, z_{n, n}$ are the zeros of $P_{n}$ (counting multiplicities) and $\delta_{z}$ denotes the unit point measure at $z$.

A sequence of measures $\left\{v_{n}\right\}_{n \geq 1}$ is said to converge in the weak*-topology to the measure $v$ (symbolically, $v_{n} \stackrel{*}{\longrightarrow} v$ as $\left.n \rightarrow \infty\right)$ if for every continuous function $f$ defined on $\overline{\mathbb{C}}, \lim _{n \rightarrow \infty} \int f d v_{n}=\int f d v$.

The equilibrium measure $\mu_{L_{\rho}}$ of $L_{\rho}$ is the probability measure supported on $L_{\rho}$ whose value at any given Borel set $B \subset L_{\rho}$ is

$$
\mu_{L_{\rho}}(B)=\frac{1}{2 \pi \rho} \int_{B^{-1}}|d t|, \quad B^{-1}:=\left\{t \in \mathbb{T}_{\rho}: \psi(t) \in B\right\} .
$$

Finally, for $\epsilon>0$ and $t \in \mathbb{C}, D_{\epsilon}(t)$ denotes the open disk with center at $t$ and radius $\epsilon$.

From Theorem 2.5(a) and the maximum modulus principle for analytic functions we see that

$$
\frac{P_{n}(z)}{\sqrt{n+1}[\phi(z)]^{n}}=\phi^{\prime}(z)+\left\{\begin{array}{c}
\mathcal{O}\left(n^{-\lambda_{1}-1}(\rho / r)^{n}\right), \\
\mathcal{O}\left(n^{-\lambda_{1}-1}\right), \quad z \in \bar{\Omega}_{\rho} \backslash \bar{\Omega}_{k=1}^{s}, \rho<r<1, \\
D_{\epsilon}\left(z_{k}\right), \epsilon>0 .
\end{array}\right.
$$

Hence, we trivially have

Corollary 2.7. For every $\epsilon>0$, there is $N_{\epsilon}>0$ such that if $n>N_{\epsilon}$, then $P_{n}(z)$ has no zeros on $\bar{\Omega}_{\rho} \backslash \cup_{k=1}^{s} D_{\epsilon}\left(z_{k}\right)$. In particular, $\mathcal{Z} \cap \Omega_{\rho}=\emptyset$.

To describe the zero behavior of $P_{n}$ inside $G_{\rho}$, it is convenient to rewrite (15) as follows. Put

$$
\hat{A}_{k}:=A_{k} e^{i\left(\lambda_{1}+1\right)\left(\Theta_{k}-\Theta_{1}\right)}, \quad 1 \leq k \leq u,
$$

and let $\theta_{1}, \theta_{2}, \ldots, \theta_{u}$ be such that

$$
e^{2 \pi i \theta_{k}}=e^{i\left(\Theta_{k}-\Theta_{1}\right)}, \quad \theta_{k} \in(0,1], \quad 1 \leq k \leq u,
$$

so that (15) takes the form

$$
P_{n}^{*}(z)=H_{n}(z)+o(1)
$$

locally uniformly on $G_{\rho}$ as $n \rightarrow \infty$, where

$$
P_{n}^{*}(z):=\frac{-P_{n}(z)}{\sqrt{n+1}\left(\begin{array}{c}
n \\
-\lambda_{1}-1
\end{array}\right)\left(\omega_{1}\right)^{n+1+\lambda_{1}}}, \quad H_{n}(z):=\varphi^{\prime}(z) \sum_{k=1}^{u} \frac{\varphi^{\prime}\left(z_{k}\right) \hat{A}_{k} e^{2 \pi i n \theta_{k}}}{\left[\varphi(z)-\varphi\left(z_{k}\right)\right]^{2}} .
$$

The zeros of $P_{n}$ coincide, of course, with those of $P_{n}^{*}$, and in view of Hurwitz theorem, $\mathcal{Z}$ contains the zeros lying in $G_{\rho}$ of those not identically zero functions that are the uniform limit of some subsequence of $\left\{H_{n}\right\}_{n \geq 0}$. We then pass to establish the general form of any uniform limit point of $\left\{H_{n}\right\}_{n \geq 0}$.

Among the numbers $1=\theta_{1}, \theta_{2}, \ldots, \theta_{u}$, there is a basis over the rationals containing $\theta_{1}$ [2, Ch. III. p. 4], say $\theta_{1}, \theta_{2}, \ldots, \theta_{u^{*}}, 1 \leq u^{*} \leq u$, so that for every $k \in\{1,2, \ldots, u\}$, there are unique rational numbers $r_{k 1}, r_{k 2}, \ldots, r_{k u^{*}}$ with

$$
\theta_{k}=\sum_{j=1}^{u^{*}} r_{k j} \theta_{j}, \quad 1 \leq k \leq u
$$

Note that $u^{*}=1$ if and only if all the $\theta_{k}$ 's are rational, and if $u^{*} \geq 2$, then $\theta_{2}, \ldots, \theta_{u^{*}}$ are irrational numbers linearly independent over the rationals.

For every $k \in\{1,2, \ldots, u\}$, let $1 \leq p_{k} \leq q_{k}$ be the unique relatively prime integers such that

$$
e^{2 \pi i r_{k 1}}=e^{2 \pi i p_{k} / q_{k}}
$$

so that

$$
e^{2 \pi i \theta_{k}}=e^{2 \pi i\left(\frac{p_{k}}{q_{k}}+\sum_{j=2}^{u^{*}} r_{k j} \theta_{j}\right)}, \quad 1 \leq k \leq u,
$$

where in case $u^{*}=1$, the sum $\sum_{j=2}^{u^{*}} \cdots$ above is understood to be zero (observe that $p_{1}=q_{1}=1$, but $p_{k}<q_{k}$ for $k>1$ ). 
Let $\mathbf{q}$ be the least common multiple of the denominators $q_{1}, q_{2}, \ldots, q_{u}$, and for every $\ell \in\{1,2, \ldots, \mathbf{q}\}$, let

$$
\ell p_{k}=s_{k \ell} \bmod q_{k}, \quad 0 \leq s_{k \ell}<q_{k} .
$$

Observe that two $u$-tuples $\left(s_{1 \ell}, s_{2 \ell}, \ldots, s_{u \ell}\right)$ corresponding to different values of $\ell$ are distinct.

Theorem 2.8. The functions $f$ that are the uniform limit of some subsequence of $\left\{H_{n}\right\}_{n \geq 0}$ are the functions of the form

$$
f(z)=\varphi^{\prime}(z) \sum_{k=1}^{u} \frac{\varphi^{\prime}\left(z_{k}\right) \hat{A}_{k} e^{2 \pi i\left(\frac{s_{k \ell}}{q_{k}}+\sum_{j=2}^{u^{*}} r_{k j} \alpha_{j}\right)}}{\left[\varphi(z)-\varphi\left(z_{k}\right)\right]^{2}}
$$

with $\ell \in\{1,2, \ldots, \mathbf{q}\}$ and $\alpha_{2}, \ldots, \alpha_{u^{*}}$ arbitrary real numbers. In particular, there is always such a limit function $f$ that is not identically zero.

Corollary 2.9. There exists a subsequence $\left\{n_{j}\right\}_{j=1}^{\infty} \subset \mathbb{N}$ such that $\nu_{n_{j}} \stackrel{*}{\longrightarrow} \mu_{L_{\rho}}$ as $j \rightarrow \infty$. Hence, $L_{\rho} \subset \mathcal{Z}$.

However, because the $z_{k}$ 's are not necessarily pairwise distinct, some of the limit functions (25) can be identically zero, which makes Theorem 2.4 insufficient to describe $\mathcal{Z} \cap G_{\rho}$. A necessary condition for this to happen is that for every $1 \leq j \leq u$,

$$
2 \max _{k: z_{k}=z_{j}}\left|A_{k}\right| \leq \sum_{k: z_{k}=z_{j}}\left|A_{k}\right|
$$

It is not sufficient though, and whether for a concrete instance of a curve $L_{\rho}$ satisfying A.1 and A.2 there will be a limit function of the form (25) that is identically zero ultimately depends on the specific values of the $\theta_{k}$ 's and can be determined, in principle, from the general form given in (25).

Let us then make the assumption that

A.3: No limit function of the form (25) is identically zero.

Such an assumption is satisfied in a large number of cases. For instance, if there is $k$ such that $z_{j} \neq z_{k}$ whenever $j \neq k$, as is the case in which $L_{\rho}$ is, in addition, a Jordan curve.

Corollary 2.10. Assume A.3 holds.

(a) The point $t \in G_{\rho}$ also belongs to $\mathcal{Z}$ if and only if there exist an integer $\ell \in\{1,2, \ldots, \mathbf{q}\}$ and real numbers $\alpha_{2}, \ldots, \alpha_{u^{*}}$ such that

$$
\sum_{k=1}^{u} \frac{\varphi^{\prime}\left(z_{k}\right) \hat{A}_{k} e^{2 \pi i\left(\frac{s_{k \ell}}{q_{k}}+\sum_{j=2}^{u^{*}} r_{k j} \alpha_{j}\right)}}{\left[\varphi(t)-\varphi\left(z_{k}\right)\right]^{2}}=0 .
$$

(b) For every compact set $E \subset G_{\rho}$ there is a number $N_{E}$ such that when $n>N_{E}$, $P_{n}$ has at most $2(J-1)$ zeros in $E$ (counting multiplicities), where $J$ is the number of corners $z_{k}$. As a consequence, $\nu_{n} \stackrel{*}{\longrightarrow} \mu_{L_{\rho}}$ as $n \rightarrow \infty$.

Remark 2.11. Assume A.3 holds, so that by Corollary 2.10(a) we have the following. If $z_{1}=z_{2}=\cdots=z_{u}$, then $\mathcal{Z} \cap G_{\rho}=\emptyset$. Otherwise:

(a) if $u^{*}=1$ (i.e., all the $\theta_{j}$ 's are rational), then the number of points in $\mathcal{Z} \cap G_{\rho}$ is finite, namely at most $2(u-1) \mathbf{q}$

(b) if $u^{*}=2$, then by fixing $\ell$ and letting $\alpha_{2}$ vary, equation (26) can be written as

$$
g_{0, \ell}(z)+g_{1, \ell}(z) w+\cdots+g_{u-1, \ell}(z) w^{2(u-1)}=0, \quad|z|=1, \quad 1 \leq \ell \leq \mathbf{q},
$$

where $w=\varphi(t)$ and the $g_{k, \ell}(z)$ 's are certain polynomials, so that if $f_{1}, \ldots, f_{m}$ are those algebraic functions that are a solution to at least one of the algebraic equations (27) (see e.g., [5, Chap. 5]), then $\mathcal{Z} \cap G_{\rho}$ consists of the traces left in $G_{\rho}$ by the curves $\varphi^{-1} \circ f_{1}\left(\mathbb{T}_{1}\right), \ldots, \varphi^{-1} \circ f_{m}\left(\mathbb{T}_{1}\right)$, plus possibly the preimages by $\varphi$ of some of the solution points corresponding to the algebraic singularities of the $f_{k}$ 's;

(c) if $u^{*} \geq 2$, then $\mathcal{Z} \cap G_{\rho}$ is, in general, a two dimensional domain.

Remark 2.12. Under Assumption A.3, finer results similar to Thm. 4 of [9] (see also [17, Thms. 11.1, 11.2]) on the separation, distribution and speed of convergence to $L_{\rho}$ of those zeros of $P_{n}$ that lie near $L_{\rho}$ but separated from the corners can be derived from Theorem 2.5(a). 
2.3. The case of some special lemniscates. In this section we consider an example where Theorem 2.4 fails to describe the behavior of certain subsequences of $\left\{P_{n}\right\}_{n \geq 0}$. In particular, it shows that Corollary 2.10(b) does not necessarily hold in the absence of Condition A.3.

Let $s \geq 2$ be a given integer. If we agree in that

$$
2 \pi(k-1) \leq \arg \left(w^{s}+1\right)<2 \pi k \quad \text { whenever } 2 \pi(k-1) / s \leq \arg (w)<2 \pi k / s,
$$

then the function $w \mapsto\left(w^{s}+1\right)^{1 / s}$ maps $\mathbb{E}_{1}$ conformally onto the exterior of the lemniscate of $s$ petals $\left\{z:\left|z^{s}-1\right|=1\right\}$ (see Figure 2 below for $s=3$ ).

Let $R>1$ be a number that has been fixed, and let

$$
L_{1}:=\left\{z:\left|z^{s}-1\right|=R^{s}\right\}=\left\{z=\left(w^{s}+1\right)^{1 / s}:|w|=R\right\} .
$$

Then, for this $L_{1}$ we have

$$
\begin{gathered}
\psi(w)=\left(R^{s} w^{s}+1\right)^{1 / s}, \quad \phi(z)=R^{-1}\left(z^{s}-1\right)^{1 / s}, \\
\rho=R^{-1}, \quad \Omega_{\rho}=\left\{z:\left|z^{s}-1\right|>1\right\}, \quad L_{\rho}=\left\{z:\left|z^{s}-1\right|=1\right\}, \quad G_{\rho}=\left\{z:\left|z^{s}-1\right|<1\right\},
\end{gathered}
$$

and it is easily seen that $\psi$ satisfies Conditions A.1 and A.2 of Subsection 2.1 with

$$
\omega_{k}=R^{-1} e^{i(2 k-1) \pi / s}, \quad z_{k}=0, \quad 0<\lambda_{k}=1 / s \leq 1 / 2, \quad k=1,2, \ldots, s .
$$

The important feature to note of this example is that the function $H_{n}$ defined in (23) is identically zero for every $n \neq s-2 \bmod s$.

Theorem 2.13. Let $\left\{P_{n}\right\}_{n=0}^{\infty}$ be the sequence of polynomials orthonormal over the interior of the lemniscate $L_{1}=\left\{z:\left|z^{s}-1\right|=R^{s}\right\}$. Then,

(a) for all $n=s m+s-1$ with $m \geq 0$ an integer,

$$
P_{n}(z)=\sqrt{n+1} R^{-(n+1)} z^{s-1}\left(z^{s}-1\right)^{m}
$$

(b) for all $n=s m+l$ with $m \geq 0$ and $0 \leq l \leq s-2$ integers,

$$
\frac{(-1)^{m} R^{n+1} \Gamma(n+(3 s-l-1) / s) P_{n}(z)}{n ! \sqrt{n+1}}=\frac{s^{(2 s-l-1) / s}}{(s-l-1) ! \Gamma((1+l-s) / s)} \frac{\partial^{s-l-2} L}{\partial \zeta^{s-l-2}}(0, z)+R_{n}(z), \quad z \in G_{\rho},
$$

where $R_{n}(z)=\mathcal{O}\left(n^{-1}\right)$ locally uniformly on $G_{\rho}$ as $n \rightarrow \infty, n \neq s-1 \bmod s$. More precisely,

$$
n R_{n}(z)=\frac{s^{(s-l-1) / s}}{\Gamma((1+l-2 s) / s)}\left[\frac{(s-1)}{2(s-l-2) !} \frac{\partial^{s-l-2} L}{\partial \zeta^{s-l-2}}(0, z)-\frac{s^{2}}{(2 s-l-2) !} \frac{\partial^{2 s-l-2} L}{\partial \zeta^{2 s-l-2}}(0, z)\right]+\mathcal{O}\left(\frac{1}{n}\right) .
$$

The partial derivatives of the function $L(\zeta, z)$ occurring in Theorem 2.13 can be explicitly computed. Indeed, it is easy to see that

$$
\varphi(z)=\frac{R z}{\left[R^{2 s}-1+z^{s}\right]^{1 / s}}
$$

maps $G_{1}$ conformally onto $\mathbb{D}_{1}$. We have $\varphi(0)=0, \varphi^{\prime}(0)=R\left(R^{2 s}-1\right)^{-1 / s}>0$, and hence

$$
L(\zeta, z)=\frac{\left(R^{2 s}-1\right)^{2}\left[R^{2 s}-1+z^{s}\right]^{(1-s) / s}\left[R^{2 s}-1+\zeta^{s}\right]^{(1-s) / s}}{\left(\left[R^{2 s}-1+z^{s}\right]^{1 / s} \zeta-z\left[R^{2 s}-1+\zeta^{s}\right]^{1 / s}\right)^{2}} .
$$

Moreover, it easily follows by mathematical induction that for all $0 \leq j \leq s-1$,

$$
\frac{\partial^{j} L(\zeta, z)}{\partial \zeta^{j}}=\frac{(-1)^{j}(j+1) !\left(R^{2 s}-1\right)^{2}\left[R^{2 s}-1+z^{s}\right]^{(1-s+j) / s}\left[R^{2 s}-1+\zeta^{s}\right]^{(1-s) / s}}{\left(\left[R^{2 s}-1+z^{s}\right]^{1 / s} \zeta-z\left[R^{2 s}-1+\zeta^{s}\right]^{1 / s}\right)^{j+2}}+\zeta^{s-j} f_{j}(\zeta, z),
$$

where $f_{j}(\cdot, z)$ is analytic at 0 , and therefore, for all $0 \leq l \leq s-2$,

$$
\frac{\partial^{s-l-2} L}{\partial \zeta^{s-l-2}}(0, z)=(s-l+1) ! z^{l-s}\left[\frac{R^{2 s}-1}{R^{2 s}-1+z^{s}}\right]^{(l+1) / s} .
$$

Thus, we obtain from Theorem 2.13(b) and (29) the following

Corollary 2.14. For any compact set $F \subset G_{\rho}$, there is a number $N_{F}$ such that if $n>N_{F}$ and $n \neq s-1$ $\bmod s$, then $P_{n}$ has no zeros on $F$. As a consequence, $\nu_{n} \stackrel{*}{\longrightarrow} \mu_{L_{\rho}}$ as $n \rightarrow \infty, n \neq s-1 \bmod s$. 


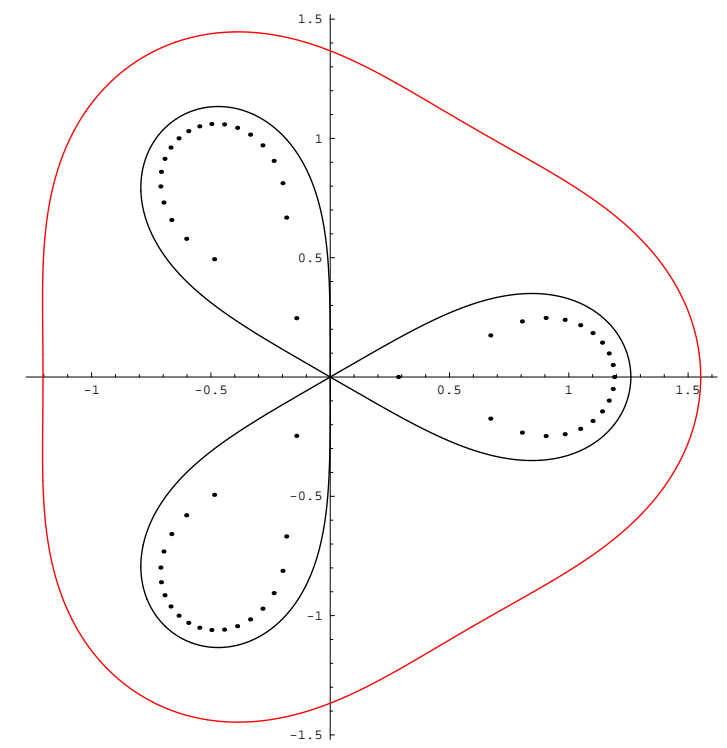

Figure 2. Zeros of $P_{60}(z)$ for $L_{1}=\left\{z:\left|z^{3}-1\right|=(1.4)^{3}\right\}$.

However, by Theorem 2.13 (a), the zeros of $P_{s m+s-1}(z)$ are fixed, namely, a zero of multiplicity $s-1$ at the origin and the points $\alpha_{k}:=e^{2 \pi i k / s}, 1 \leq k \leq s$, each of multiplicity $m$ and contained in $G_{\rho}$. Therefore,

$$
\nu_{s m+s-1} \stackrel{*}{\longrightarrow} \frac{1}{s} \sum_{k=1}^{s} \delta_{\alpha_{k}} \quad \text { as } m \rightarrow \infty .
$$

Thus, Corollary 2.10(b) does not necessarily hold in the absence of Condition A.3.

The assertion in Corollary 2.14 concerning the convergence of the measures $\nu_{n}$ can be proven by employing the exact same argument used in the proof of Corollary 2.10(b).

\section{ProOfs}

3.1. Proofs of Theorem $\mathbf{2 . 2}$ and Corollary 2.3. We first make a short remark about the estimates in (51). Carleman stated his formula (41) [1, Satz IV] as holding uniformly on the exterior of the curve $L_{1}$ with the estimate $h_{n}(z)=\mathcal{O}\left(\sqrt{n} \rho^{n}\right)$. However, as shown by Gaier in [4, Thm. 2, §2], without any variation, Carleman's proof equally yields that (4) also holds uniformly on any $L_{r}, \rho<r<1$, with the estimate $h_{n}(z)=\mathcal{O}\left(n^{-1 / 2}(\rho / r)^{n}\right)$. Here we note that, indeed, from Carleman's proof it actually follows that on any $L_{r}$ with $1<r<\infty$, (4) holds with the estimate $h_{n}(z)=\mathcal{O}\left(\rho^{n}\right)$. To see this, follow Gaier's presentation [4, p. 13] of Carleman's proof, and notice that, in Gaier's notation,

$$
\sum_{j=1}^{n} j \rho^{2 j-2-2 n}= \begin{cases}n(n+1) / 2, & \rho=1 \\ \frac{n \rho^{2 n}\left(\rho^{2}-1\right)+1-\rho^{2 n}}{\rho^{2 n}\left(1-\rho^{2}\right)^{2}}, & \rho \neq 1\end{cases}
$$

(beware that the meanings of $\rho$ and $r$ in the present paper are exchanged in Gaier's presentation) so that the quantity $C_{n}$ defined in page 15 of [4] indeed satisfies

$$
C_{n}= \begin{cases}\mathcal{O}\left(r^{n}\right), & \rho>1 \\ \mathcal{O}\left(\sqrt{n} r^{n}\right), & \rho=1, \\ \mathcal{O}\left(n^{-1 / 2}(r / \rho)^{n}\right), & r<\rho<1,\end{cases}
$$

from which one gets (5) by following the same line of argument that concludes the proof in [4].

Proof of Theorem 2.2. Equality (11) holds uniformly in $\zeta \in \bar{G}_{1}$ for each fixed $z \in G_{1}$. We then replace $P_{k}(\zeta)$ in (11) by its corresponding asymptotic representation given by the right-hand side of (4)), multiply 
the resulting equality by $[\phi(\zeta)]^{-(n+1)} / 2 \pi i$ and integrate it over $L_{1}$ to obtain

$$
\frac{\overline{\varphi^{\prime}(z)}}{2 \pi i} \oint_{L_{1}} \frac{\varphi^{\prime}(\zeta)[\phi(\zeta)]^{-(n+1)} d \zeta}{[1-\overline{\varphi(z)} \varphi(\zeta)]^{2}}=\sum_{k=0}^{\infty} \frac{\overline{P_{k}(z)} \sqrt{k+1}}{2 \pi i} \oint_{L_{1}} \phi^{\prime}(\zeta)[\phi(\zeta)]^{k-n-1}\left[1+h_{k}(\zeta)\right] d \zeta .
$$

On the one hand, we have

$$
d \zeta=\frac{i \varphi(\zeta)\left|\varphi^{\prime}(\zeta)\right||d \zeta|}{\varphi^{\prime}(\zeta)} \Rightarrow \overline{d \zeta}=-\frac{\varphi^{\prime}(\zeta) d \zeta}{\overline{\varphi^{\prime}(\zeta)}[\varphi(\zeta)]^{2}},
$$

so that

$$
\overline{\oint_{L_{1}} \frac{\varphi^{\prime}(\zeta)[\phi(\zeta)]^{-(n+1)} d \zeta}{[1-\overline{\varphi(z)} \varphi(\zeta)]^{2}}}=-\oint_{L_{1}} \frac{\varphi^{\prime}(\zeta)[\phi(\zeta)]^{n+1} d \zeta}{[\varphi(\zeta)-\varphi(z)]^{2}}
$$

On the other hand,

$$
\frac{1}{2 \pi i} \oint_{L_{1}} \phi^{\prime}(\zeta)[\phi(\zeta)]^{k-n-1} d \zeta=\frac{1}{2 \pi i} \oint_{\mathbb{T}_{1}} t^{k-n-1} d t=\left\{\begin{array}{ll}
0, & \text { if } n \neq k, \\
1, & \text { if } n=k,
\end{array} \quad k, n \geq 0,\right.
$$

and since $h_{k}(\psi(w))$ is analytic in $\mathbb{E}_{\rho}$,

$$
\frac{1}{2 \pi i} \oint_{L_{1}} \phi^{\prime}(\zeta)[\phi(\zeta)]^{k-n-1} h_{k}(\zeta) d \zeta=\frac{1}{2 \pi i} \oint_{\mathbb{T}_{1}} t^{k-n-1} h_{k}(\psi(t)) d t=0, \quad 0 \leq k \leq n-1 .
$$

Therefore, we get from (30), (31) and the two previous relations that

$$
\frac{\varphi^{\prime}(z)}{\sqrt{n+1} 2 \pi i} \oint_{L_{1}} \frac{\varphi^{\prime}(\zeta)[\phi(\zeta)]^{n+1} d \zeta}{[\varphi(\zeta)-\varphi(z)]^{2}}=P_{n}(z)-\epsilon_{n}(z), \quad z \in G_{1},
$$

where

$$
\epsilon_{n}(z)=-\sum_{j=0}^{\infty} \sqrt{1+j /(n+1)} \alpha_{j, n} P_{n+j}(z), \quad z \in G_{1}
$$

with

$$
\alpha_{j, n}:=\overline{\frac{1}{2 \pi i} \oint_{L_{1}} \phi^{\prime}(\zeta)[\phi(\zeta)]^{j-1} h_{n+j}(\zeta) d \zeta} .
$$

Now, from (5) we obtain that for every $1<\eta<\infty$, there is a constant $M_{\eta}$ that only depends on $\eta$, such that for all integers $j, n \geq 0$,

$$
\left|\alpha_{j, n}\right|=\left|\frac{1}{2 \pi i} \oint_{L_{\eta}} \phi^{\prime}(\zeta)[\phi(\zeta)]^{j-1} h_{n+j}(\zeta) d \zeta\right| \leq M_{\eta} \eta^{j} \rho^{n+j}
$$

By Carleman's formula, for every $r \in(\rho, 1 / \rho)$, there is some constant $K_{r}$ such that $\left|P_{n}(z)\right| \leq K_{r} \sqrt{n+1} r^{n}$ for all $z \in \bar{G}_{r}$, so that for $1<\eta<(\rho r)^{-1}$

$$
\begin{aligned}
\sum_{j=0}^{\infty} \sqrt{1+j /(n+1)}\left|\overline{\alpha_{j, n}} P_{n+j}(z)\right| & \leq \sqrt{n+1}(r \rho)^{n} M_{\eta} K_{r} \sum_{j=0}^{\infty}\left(1+\frac{j}{n+1}\right)(\eta r \rho)^{j} \\
& \leq \frac{\sqrt{n+1}(r \rho)^{n} M_{\eta} K_{r}}{(1-\eta r \rho)^{2}}, \quad z \in \bar{G}_{r} .
\end{aligned}
$$

This shows that the series in the right-hand side of (33) that defines $\epsilon_{n}(z)$ for $z \in G_{1}$, indeed converges locally uniformly on $G_{1 / \rho}$ to an analytic function (the analytic continuation of $\epsilon_{n}(z)$ ). In the same way one sees that if for some $\tau \in[0,1 / \rho)$ and constant $K_{\tau},\left|P_{n}(z)\right| \leq K_{\tau} \sqrt{n+1} \tau^{n}$ for all $z \in E \subset G_{1 / \rho}$, then for any fixed $1<\eta<(\rho \tau)^{-1}$,

$$
\left|\epsilon_{n}(z)\right| \leq \frac{\sqrt{n+1}(\tau \rho)^{n} M_{\eta} K_{\tau}}{(1-\eta \tau \rho)^{2}} \quad \forall z \in E .
$$

The proof of Theorem 2.2 is complete. 
Proof of Corollary 2.3. Equality (14) holds for any given conformal map $\varphi$ of $G_{1}$ onto $\mathbb{D}_{1}$. Let us pick specifically a map $\varphi$ such that $\varphi(z) \neq 0$ for all $z \in G_{1} \cap \Omega_{\rho}$. Such a map has an analytic and univalent continuation to all of $G_{1 / \rho}$ (given by (9) and (10) ), so that for arbitrary $\eta \in(1,1 / \rho)$,

$$
P_{n}(z)=\frac{\sqrt{n+1} \varphi^{\prime}(z)}{2 \pi i} \oint_{L_{\eta}} \frac{\phi^{\prime}(\zeta)[\phi(\zeta)]^{n} d \zeta}{\varphi(\zeta)-\varphi(z)}+\epsilon_{n}(z), \quad z \in G_{1} .
$$

The right-hand side of (35) is indeed a well-defined analytic function (in the variable $z$ ) on $G_{\eta}$, so that by analytic continuation, (35) actually holds for all $z \in G_{\eta}$. Now, for every $z \in L_{1}$, the function (in the variable $\zeta) \varphi^{\prime}(z) /[\varphi(\zeta)-\varphi(z)]$ is analytic at all points of $G_{1 / \rho} \cap \Omega_{\rho}$, with the exception of the point $z$, where it has a simple pole of residue 1 . From the residue theorem, it follows that for every $r \in(\rho, 1)$,

$$
P_{n}(z)=\sqrt{n+1} \phi^{\prime}(z)[\phi(z)]^{n}+\frac{\sqrt{n+1} \varphi^{\prime}(z)}{2 \pi i} \oint_{L_{r}} \frac{\phi^{\prime}(\zeta)[\phi(\zeta)]^{n} d \zeta}{\varphi(\zeta)-\varphi(z)}+\epsilon_{n}(z), \quad z \in L_{1} .
$$

Now, fix $r^{\prime} \in(\rho, 1)$, so that $L_{r}$ is contained in the interior of $L_{r^{\prime}}$ whenever $\rho<r<r^{\prime}$, and therefore

$$
\begin{aligned}
\left|\frac{1}{2 \pi i} \oint_{L_{r}} \frac{\phi^{\prime}(\zeta)[\phi(\zeta)]^{n} d \zeta}{\varphi(\zeta)-\varphi(z)}\right| & =\left|\frac{1}{2 \pi i} \oint_{\mathbb{T}_{r}} \frac{t^{n} d t}{\varphi(\psi(t))-\varphi(z)}\right| \leq \frac{r^{n+1}}{\operatorname{dist}\left(\mathbb{T}_{1}, \varphi\left(L_{r}\right)\right)} \\
& \leq \frac{r^{n+1}}{\operatorname{dist}\left(\mathbb{T}_{1}, \varphi\left(L_{r^{\prime}}\right)\right)}, \quad \rho<r<r^{\prime}, \quad z \in L_{1} .
\end{aligned}
$$

Since by Theorem 2.2, we have

$$
P_{n}(z)=\mathcal{O}(\sqrt{n}) \Rightarrow e_{n}(z)=\mathcal{O}\left(\sqrt{n} \rho^{n}\right), \quad z \in L_{1},
$$

it follows from (36) and (37) by letting $r \rightarrow \rho$ that

$$
P_{n}(z)=\sqrt{n+1} \phi^{\prime}(z)[\phi(z)]^{n}+\mathcal{O}\left(\sqrt{n} \rho^{n}\right)=\sqrt{n+1} \phi^{\prime}(z)[\phi(z)]^{n}\left[1+\mathcal{O}\left(\rho^{n}\right)\right], \quad z \in L_{1} .
$$

The proof of Corollary 2.3 is complete.

3.2. Asymptotic expansions. Through the remaining of this paper, we assume that the map $\psi$ satisfies Conditions A.1 and A.2 stated in Subsection 2.1 .

Let $T_{k}$ be a small open circular arc of $\mathbb{T}_{\rho}$ centered at $\omega_{k}$ such that $\overline{T_{k}} \cap\left\{\omega_{1}, \ldots, \omega_{s}\right\}=\left\{\omega_{k}\right\}$. The set $T_{k} \backslash\left\{\omega_{k}\right\}$ consists of two circular arcs, say $T_{k}^{+}, T_{k}^{-}$, and by our Assumption A.1 on $L_{\rho}$, there exist simple analytic $\operatorname{arcs} \mathcal{L}_{k}^{+} \supset \psi\left(\overline{T_{k}^{+}}\right)$and $\mathcal{L}_{k}^{-} \supset \psi\left(\overline{T_{k}^{-}}\right)$of which $z_{k}$ is an interior point. Hence, the map $\psi$, originally defined on $\mathbb{E}_{\rho}$, can be continued by the Schwarz reflection principle for analytic arcs [3] across both $T_{k}^{+}$and $T_{k}^{-}$. Since the images of $\mathcal{L}_{k}^{+}$and $\mathcal{L}_{k}^{-}$in such reflections are again simple analytic arcs containing $z_{k}$ as an interior point, by applying subsequent reflections we can continue $\psi$ near $\omega_{k}$ onto the entire logarithmic Riemann surface $\mathcal{S}_{\omega_{k}}$ with branch point at $\omega_{k}$.

Let the functions $\left(w-\omega_{k}\right)^{l+j \lambda_{k}}, l \geq 0, j \geq 1$, and $\log \left(w-\omega_{k}\right)$ be defined in $\mathcal{S}_{\omega_{k}}$. In what follows we abbreviate by putting $y=w-\omega_{k}$. Lehman [7, Thm. 1] proved that when $\lambda_{k}>0$, $\psi$ has the following asymptotic expansion about $\omega_{k}$ : if $\lambda_{k}$ is irrational, then

$$
\psi(w)=\psi\left(\omega_{k}\right)+\sum_{l=0}^{\infty} \sum_{j=1}^{\infty} c_{l j 0}^{k} y^{l+j \lambda_{k}}, \quad c_{010}^{k} \neq 0 ;
$$

if $\lambda_{k}=p / q$ is a fraction reduced to lowest terms, then

$$
\psi(w)=\psi\left(\omega_{k}\right)+\sum_{l=0}^{\infty} \sum_{j=1}^{q} \sum_{m=0}^{\lfloor l / p\rfloor} c_{l j m}^{k} y^{l+j \lambda_{k}}(\log y)^{m}, \quad c_{010}^{k} \neq 0 .
$$

The terms in the above series are assumed to be arranged in an order such that a term of the form $y^{l+j \lambda_{k}}(\log y)^{m}$ precedes one of the form $y^{l^{\prime}+j^{\prime} \lambda_{k}}(\log y)^{m^{\prime}}$ if either $l+j \lambda_{k}<l^{\prime}+j^{\prime} \lambda_{k}$ or $l+j \lambda_{k}=l^{\prime}+j^{\prime} \lambda_{k}$ and $m>m^{\prime}$. We write in (38) $c_{l j 0}^{k}$ instead of simply $c_{l j}^{k}$ when $\lambda_{k}$ is irrational, because this will allow us to express many of the relations that follow in one single statement without having to distinguish between $\lambda_{k}$ being irrational or rational. 
The precise meaning of these expansions is the following: if according to the order explained above, (38) and (39) are written in the form

$$
\psi(w)=\psi\left(\omega_{k}\right)+\sum_{n=1}^{\infty} \chi_{n}(y)
$$

then for all $N \geq 1$,

$$
\psi(w)-\psi\left(\omega_{k}\right)-\sum_{n=1}^{N} \chi_{n}(y)=o\left(\chi_{N}(y)\right)
$$

as $w \rightarrow \omega_{k}(y \rightarrow 0)$ from any finite sector $\vartheta_{1} \leq \arg \left(w-\omega_{k}\right) \leq \vartheta_{2}$ of $\mathcal{S}_{\omega_{k}}$.

The coefficients $c_{l j m}^{k}$ in (38) and (39) depend on the values assigned to the functions $\left(w-\omega_{k}\right)^{l+j \lambda_{k}}$, $\log \left(w-\omega_{k}\right)$ at a specified point of $\mathcal{S}_{\omega_{k}}$. We shall assume that the values of $\psi$ in $\mathbb{E}_{\rho}$ define $\psi$ in the sector $\Theta_{k}-\pi<\arg \left(w-\omega_{k}\right)<\Theta_{k}+\pi$ of $\mathcal{S}_{\omega_{k}}$, and that for every $w$ in this sector,

$$
(y)^{l+j \lambda_{k}}=|y|^{l+j \lambda_{k}} e^{i\left(l+j \lambda_{k}\right) \arg (y)}, \quad \log y=\log |y|+i \arg (y), \quad y=w-\omega_{k} .
$$

Let

$$
A_{k}:=c_{010}^{k}=\lim _{\substack{w \rightarrow \omega_{k} \\ w \in \mathbb{E}_{\rho}}} \frac{\psi(w)-\psi\left(\omega_{k}\right)}{\left(w-\omega_{k}\right)^{\lambda_{k}}}(\neq 0), \quad 1 \leq k \leq s,
$$

so that the following relations follow from (38) and (39). If $1 / 2<\lambda_{k}<1$, then

$$
\psi(w)=\psi\left(\omega_{k}\right)+A_{k} y^{\lambda_{k}}+c_{020}^{k} y^{2 \lambda_{k}}+c_{110}^{k} y^{1+\lambda_{k}}+\mathcal{O}\left(y^{3 \lambda_{k}}\right)
$$

if $\lambda_{k}=1 / 2$, then

$$
\psi(w)=\psi\left(\omega_{k}\right)+A_{k} y^{\lambda_{k}}+c_{020}^{k} y^{2 \lambda_{k}}+c_{111}^{k} y^{1+\lambda_{k}} \log y+\mathcal{O}\left(y^{3 \lambda_{k}}\right) ;
$$

if $0<\lambda_{k}<1 / 2$ and $v_{k}$ is sufficiently small (say, $0<v_{k}<\min \left\{\lambda_{k}, 1-2 \lambda_{k}\right\}$ ), then

$$
\psi(w)=\psi\left(\omega_{k}\right)+A_{k} y^{\lambda_{k}}+c_{020}^{k} y^{2 \lambda_{k}}+c_{030}^{k} y^{3 \lambda_{k}}+o\left(y^{3 \lambda_{k}+v_{k}}\right) ;
$$

if $1<\lambda_{k}<2$, then

$$
\psi(w)=\psi\left(\omega_{k}\right)+A_{k} y^{\lambda_{k}}+c_{110}^{k} y^{1+\lambda_{k}}+c_{020}^{k} y^{2 \lambda_{k}}+\mathcal{O}\left(y^{2+\lambda_{k}}\right)
$$

(notice that if $1<\lambda_{k}=p / q<2$, then $p \geq 3, q \geq 2$, and no log-terms correspond to $l=0,1,2$ ).

We analyze now the behavior of $\varphi^{\prime}(z) /[\varphi(\psi(w))-\varphi(z)]$ as $w \rightarrow \omega_{k}$, where $\varphi$ is a conformal map of $G_{1}$ onto $\mathbb{D}_{1}$. For given $\delta>0$ and $t \in \mathbb{C}$, we put

$$
D_{\delta}(t):=\{w:|w-t|<\delta\} .
$$

We have already noticed in the introduction that the kernel

$$
L(\zeta, z):=\frac{\varphi^{\prime}(\zeta) \varphi^{\prime}(z)}{[\varphi(\zeta)-\varphi(z)]^{2}}, \quad \zeta, z \in G_{1}
$$

is independent of the map $\varphi$. For fixed $z \in G_{1}, L(\cdot, z)$ is analytic on $G_{1} \backslash\{z\}$. Hence, if $\epsilon>0$ is such that $D_{\epsilon}\left(z_{k}\right) \subset G_{1}$, then from Taylor's inequality we find that for all $\zeta \in D_{\epsilon}\left(z_{k}\right), z \in G_{1} \backslash D_{\epsilon}\left(z_{k}\right)$, and integer $N \geq 1$,

$$
\frac{\varphi^{\prime}(z)}{\varphi(\zeta)-\varphi(z)}=\frac{\varphi^{\prime}(z)}{\varphi\left(z_{k}\right)-\varphi(z)}-\sum_{j=0}^{N-1} \frac{\partial^{j} L}{\partial \zeta^{j}}\left(z_{k}, z\right) \frac{\left(\zeta-z_{k}\right)^{j+1}}{(j+1) !}+R_{N}\left(\zeta, z_{k}, z\right)
$$

with

$$
\left|R_{N}\left(\zeta, z_{k}, z\right)\right| \leq \frac{2\left|\zeta-z_{k}\right|^{N+1}}{(N+1) !} \max \left\{\left|\frac{\partial^{N} L}{\partial x^{N}}(x, z)\right|:\left|x-z_{k}\right| \leq r, z \in G_{1} \backslash D_{\epsilon}\left(z_{k}\right)\right\}, \quad\left|\zeta-z_{k}\right| \leq r<\epsilon .
$$


Combining this for $N=2$ with (40), (41), (42) and (43) gives the following: if $0<\lambda_{k}<1$, then

$$
\begin{aligned}
\frac{\varphi^{\prime}(z)}{\varphi(\psi(w))-\varphi(z)}= & \frac{\varphi^{\prime}(z)}{\varphi\left(z_{k}\right)-\varphi(z)}-L\left(z_{k}, z\right) A_{k} y^{\lambda_{k}}-\left[L\left(z_{k}, z\right) c_{020}^{k}+\frac{\partial L}{\partial \zeta}\left(z_{k}, z\right) \frac{\left(A_{k}\right)^{2}}{2}\right] y^{2 \lambda_{k}} \\
& - \begin{cases}\mathcal{O}\left(y^{1+\lambda_{k}}\right), & 1 / 2<\lambda_{k}<1, \\
\mathcal{O}\left(y^{3 \lambda_{k}}\right), & 0<\lambda_{k}<1 / 2, \\
L\left(z_{k}, z\right) c_{111}^{k} y^{3 \lambda_{k}} \log y+\mathcal{O}\left(y^{3 \lambda_{k}}\right), & \lambda_{k}=1 / 2,\end{cases}
\end{aligned}
$$

while for $1<\lambda_{k}<2$,

$$
\frac{\varphi^{\prime}(z)}{\varphi(\psi(w))-\varphi(z)}=\frac{\varphi^{\prime}(z)}{\varphi\left(z_{k}\right)-\varphi(z)}-L\left(z_{k}, z\right) A_{k} y^{\lambda_{k}}-L\left(z_{k}, z\right) c_{110}^{k} y^{1+\lambda_{k}}+\mathcal{O}\left(y^{2 \lambda_{k}}\right) .
$$

These relations hold uniformly in $z$ on compact subsets of $G_{1} \backslash\left\{z_{k}\right\}$ as $w \rightarrow \omega_{k}(y \rightarrow 0)$ from any finite sector $\vartheta_{1} \leq \arg \left(w-\omega_{k}\right) \leq \vartheta_{2}$ of $\mathcal{S}_{\omega_{k}}$. That is, if $\delta>0$ is so small that

$$
\left\{\psi(w): \vartheta_{1} \leq \arg \left(w-\omega_{k}\right) \leq \vartheta_{2},\left|w-\omega_{k}\right| \leq \delta\right\} \subset D_{\epsilon}\left(z_{k}\right)
$$

then (45) and (46)) hold uniformly for $z \in G_{1} \backslash D_{\epsilon}\left(z_{k}\right)$ and $w$ satisfying $\vartheta_{1} \leq \arg \left(w-\omega_{k}\right) \leq \vartheta_{2},\left|w-\omega_{k}\right| \leq \delta$.

Now, given $k \in\{1,2, \ldots, s\}$, the Laurent expansion of $L\left(\cdot, z_{k}\right)$ about $z_{k}$ has the form

$$
L\left(\zeta, z_{k}\right)=\frac{1}{\left(\zeta-z_{k}\right)^{2}}+a_{k, 0}+a_{k, 1}\left(\zeta-z_{k}\right)+a_{k, 2}\left(\zeta-z_{k}\right)^{2}+\cdots,
$$

with

so that

$$
a_{k, 0}=\frac{2 \varphi^{\prime}\left(z_{k}\right) \varphi^{\prime \prime \prime}\left(z_{k}\right)-3\left[\varphi^{\prime \prime}\left(z_{k}\right)\right]^{2}}{12\left[\varphi^{\prime}\left(z_{k}\right)\right]^{2}}
$$

$$
\frac{\varphi^{\prime}\left(z_{k}\right)}{\varphi(\psi(w))-\varphi\left(z_{k}\right)}=\frac{1}{\psi(w)-z_{k}}-\frac{\varphi^{\prime \prime}\left(z_{k}\right)}{2 \varphi^{\prime}\left(z_{k}\right)}-\sum_{j=0}^{\infty} \frac{a_{k, j}\left(\psi(w)-z_{k}\right)^{j+1}}{j+1}
$$

and we obtain from (47), (40), (41), (42) and (43) that if $1 / 2<\lambda_{k}<1$,

$$
\frac{\varphi^{\prime}\left(z_{k}\right)}{\varphi(\psi(w))-\varphi\left(z_{k}\right)}=\frac{1}{A_{k} y^{\lambda_{k}}}-\frac{\varphi^{\prime \prime}\left(z_{k}\right)}{2 \varphi^{\prime}\left(z_{k}\right)}-\frac{c_{020}^{k}}{\left(A_{k}\right)^{2}}-\frac{c_{110}^{k} y^{1-\lambda_{k}}}{\left(A_{k}\right)^{2}}+\mathcal{O}\left(y^{\lambda_{k}}\right)
$$

if $0<\lambda_{k}<1 / 2$ and $v_{k}$ is sufficiently small (say, $0<v_{k}<\min \left\{\lambda_{k}, 1-2 \lambda_{k}\right\}$ ), then

$$
\frac{\varphi^{\prime}\left(z_{k}\right)}{\varphi(\psi(w))-\varphi\left(z_{k}\right)}=\frac{1}{A_{k} y^{\lambda_{k}}}-\frac{\varphi^{\prime \prime}\left(z_{k}\right)}{2 \varphi^{\prime}\left(z_{k}\right)}-\frac{c_{020}^{k}}{\left(A_{k}\right)^{2}}+\left[\frac{\left(c_{020}^{k}\right)^{2}-A_{k} c_{030}^{k}-a_{k, 0}\left(A_{k}\right)^{4}}{\left(A_{k}\right)^{3}}\right] y^{\lambda_{k}}+\mathcal{O}\left(y^{\lambda_{k}+v_{k}}\right),
$$

if $\lambda_{k}=1 / 2$,

$$
\frac{\varphi^{\prime}\left(z_{k}\right)}{\varphi(\psi(w))-\varphi\left(z_{k}\right)}=\frac{1}{A_{k} y^{\lambda_{k}}}-\frac{\varphi^{\prime \prime}\left(z_{k}\right)}{2 \varphi^{\prime}\left(z_{k}\right)}-\frac{c_{020}^{k}}{\left(A_{k}\right)^{2}}+\left[\frac{\left(c_{020}^{k}\right)^{2}-a_{k, 0}\left(A_{k}\right)^{4}}{\left(A_{k}\right)^{3}}\right] y^{\lambda_{k}}+\mathcal{O}\left(y^{\lambda_{k}} \log y\right),
$$

and finally, if $1<\lambda_{k}<2$, then

$$
\frac{\varphi^{\prime}\left(z_{k}\right)}{\varphi(\psi(w))-\varphi\left(z_{k}\right)}=\frac{1}{A_{k} y^{\lambda_{k}}}-\frac{\varphi^{\prime \prime}\left(z_{k}\right)}{2 \varphi^{\prime}\left(z_{k}\right)}-\frac{c_{110}^{k} y^{1-\lambda_{k}}}{\left(A_{k}\right)^{2}}+\mathcal{O}(1)
$$

Relations (48), (49), (50) and (51) hold uniformly as $w \rightarrow \omega_{k}$ from any finite sector $\vartheta_{1} \leq \arg \left(w-\omega_{k}\right) \leq \vartheta_{2}$ of $\mathcal{S}_{\omega_{k}}$.

3.3. Auxiliary lemmas. Recall we are using the notation

$$
\mathbb{T}_{r}:=\{w:|w|=r\}, \quad \mathbb{D}_{r}:=\{w:|w|<r\}, \quad \mathbb{E}_{r}:=\{w: r<|w| \leq \infty\} .
$$

For $\delta \in(0, \rho)$ and $1 \leq k \leq s$, we have also already defined

$$
\Sigma_{\delta, k}:=\left\{w: \rho-\delta<|w|<\rho^{2} /(\rho-\delta), \Theta_{k}-\delta<\arg (w)<\Theta_{k}+\delta\right\} .
$$

Note that $\Sigma_{\delta, k}$ is the reflection of itself about the circle $\mathbb{T}_{\rho}$. Let

$$
T_{\delta, k}^{+}:=\left\{w \in \mathbb{T}_{\rho}: \Theta_{k} \leq \arg (w)<\Theta_{k}+\delta\right\}, \quad T_{\delta, k}^{-}:=\left\{w \in \mathbb{T}_{\rho}: \Theta_{k}-\delta<\arg (w) \leq \Theta_{k}\right\},
$$


and

$$
\Sigma_{\delta, k}^{+}:=\Sigma_{\delta, k} \backslash T_{\delta, k}^{+}, \quad \Sigma_{\delta, k}^{-}:=\Sigma_{\delta, k} \backslash T_{\delta, k}^{-} .
$$

For all $\delta>0$ sufficiently small, the mapping $\psi$ has analytic continuations $\psi_{+}, \psi_{-}$from $\mathbb{E}_{\rho}$ to $\Sigma_{\delta, k}^{+}, \Sigma_{\delta, k}^{-}$, respectively. To see this, fix a number $\delta^{\prime}>0$ such that $\overline{T_{\delta^{\prime}, k}^{-}} \cap\left\{\omega_{1}, \ldots, \omega_{s}\right\}=\left\{\omega_{k}\right\}$. By Assumption A.1, there is a simple analytic arc $\mathcal{L}_{k}^{-}$, of which $z_{k}$ is an interior point, such that $\mathcal{L}_{k}^{-} \supset \psi\left(T_{\delta^{\prime}, k}^{-}\right)$. Let $z^{*}$ denote the Schwarz reflection of $z$ about $\mathcal{L}_{k}^{-}$(see [3]), which is well-defined in some neighborhood $U^{k}$ of $\mathcal{L}_{k}^{-}$. Then, for every $\delta \in\left(0, \delta^{\prime}\right)$ so small that $\psi\left(\overline{\mathbb{E}}_{\rho} \cap \Sigma_{\delta, k}\right) \subset U^{k}$, the analytic continuation $\psi_{+}$of $\psi$ from $\mathbb{E}_{\rho}$ to $\Sigma_{\delta, k}^{+}$is given by

$$
\psi_{+}(w)=\left[\psi\left(\rho^{2} / \bar{w}\right)\right]^{*}, \quad w \in \Sigma_{\delta, k}^{+} \cap \mathbb{D}_{\rho} .
$$

For $a, b \in \mathbb{C}$, we denote by $[a, b]$ the oriented closed segment that starts at $a$ and ends at $b$. A similar meaning is attached to $(a, b),(a, b]$ and $[a, b)$.

For every $0<\sigma<\rho$, we define

$$
\sigma_{k}:=\sigma \omega_{k} / \rho=\sigma e^{i \Theta_{k}}, \quad 1 \leq k \leq s
$$

and the contour

$$
\Gamma_{\sigma}:=\mathbb{T}_{\sigma} \cup\left(\cup_{k=1}^{s}\left[\sigma_{k}, \omega_{k}\right]\right) .
$$

Each segment $\left[\sigma_{k}, \omega_{k}\right]$ of $\Gamma_{\sigma}$ is thought of as having two sides, and the exterior of the contour $\Gamma_{\sigma}$, denoted by $\operatorname{ext}\left(\Gamma_{\sigma}\right)$, is understood to be the unbounded component of $\overline{\mathbb{C}} \backslash \Gamma_{\sigma}$, that is,

$$
\operatorname{ext}\left(\Gamma_{\sigma}\right)=\mathbb{E}_{\sigma} \backslash\left(\cup_{k=1}^{s}\left[\sigma_{k}, \omega_{k}\right]\right) .
$$

Lemma 3.1. Let $\delta \in(0, \rho)$ be such that $\Sigma_{\delta, 1}, \Sigma_{\delta, 2}, \ldots, \Sigma_{\delta, s}$ are pairwise disjoint and $\psi$ has analytic continuations $\psi_{ \pm}$to $\Sigma_{\delta, k}^{ \pm}$for each $1 \leq k \leq s$. Then there exists $\sigma \in(\rho-\delta, \rho)$ such that

(a) $\psi$ has an analytic continuation from $\mathbb{E}_{\rho}$ to $\operatorname{ext}\left(\Gamma_{\sigma}\right)$ with continuous boundary values on $\Gamma_{\sigma}$ when viewing each $\left[\sigma_{k}, \omega_{k}\right]$ as having two sides;

(b) $\psi$ is one-to-one on $A_{\delta, \sigma}:=\overline{\mathbb{E}}_{\sigma} \backslash \cup_{k=1}^{s} \overline{\Sigma_{\delta, k}}$ and

$$
\psi\left(A_{\delta, \sigma}\right) \cap \overline{\psi\left(\operatorname{ext}\left(\Gamma_{\sigma}\right) \backslash A_{\delta, \sigma}\right)}=\emptyset .
$$

Proof. We first need to introduce the following notation. The set $\mathbb{T}_{\rho} \backslash\left\{\omega_{1}, \omega_{2}, \ldots, \omega_{s}\right\}$ consists of $s$ open circular $\operatorname{arcs} \ell^{1}, \ell^{2}, \ldots, \ell^{s}$. Let $k \in\{1,2, \ldots, s\}$, and choose numbers $\alpha_{1}<\alpha_{2}$ (which depend on $k$ ) such that

$$
\ell^{k}=\left\{\rho e^{i \alpha}: \alpha_{1}<\alpha<\alpha_{2}\right\}
$$

Then, for any $v>0$ and $\sigma \in(0, \rho)$, we define

$$
\begin{gathered}
\ell_{v}^{k}:=\left\{\rho e^{i \alpha}: \alpha_{1}+v \leq \alpha \leq \alpha_{2}-v\right\}, \\
O_{v, \sigma}^{k}:=\left\{w: \sigma<|w|<\rho^{2} / \sigma, \alpha_{1}+v<\arg (w)<\alpha_{2}-v\right\} .
\end{gathered}
$$

Observe that $\ell_{0}^{k}=\overline{\ell^{k}}$, and that $O_{v, \sigma}^{k}$ is an open set whose reflection about $\mathbb{T}_{\rho}$ coincides with itself.

Let $\delta_{1}>0$ be so small that each of the $\operatorname{arcs} \ell_{\delta_{1}}^{1}, \ell_{\delta_{1}}^{2}, \ldots, \ell_{\delta_{1}}^{s}$ has positive length. We first prove the following

Claim: for all $\sigma \in\left(\rho-\delta_{1}, \rho\right)$ sufficiently close to $\rho$, $\psi$ has an analytic continuation from $\mathbb{E}_{\rho}$ to $\mathbb{E}_{\rho} \cup$ $\left[\cup_{k=1}^{s} O_{\delta_{1}, \sigma}^{k}\right]$ which is one-to-one on $\cup_{k=1}^{s} O_{\delta_{1}, \sigma}^{k}$.

In effect, for each $1 \leq k \leq s$, the Schwarz reflection $z \mapsto z^{*}$ about the simple analytic arc $\psi\left(\ell_{\delta_{1}}^{k}\right)$ is a well-defined (one-to-one and antianalytic) function on some small neighborhood $U^{k}$ of $\psi\left(\ell_{\delta_{1}}^{k}\right)$, so that for all $\sigma$ so close to $\rho$ that $\psi\left(\overline{\mathbb{E}}_{\rho} \cap O_{\delta_{1}, \sigma}^{k}\right) \subset U^{k}$, the analytic continuation of $\psi$ to $O_{\delta_{1}, \sigma}^{k}$ is given by

$$
\psi(w)=\left[\psi\left(\rho^{2} / \bar{w}\right)\right]^{*}, \quad w \in O_{\delta_{1}, \sigma}^{k} \cap \mathbb{D}_{\rho} .
$$

By Assumption A.1, $\psi$ is one-to-one on $\overline{\mathbb{E}}_{\rho} \backslash\left\{\omega_{1}, \omega_{2}, \ldots, \omega_{s}\right\}$, and being the closed arcs $\ell_{\delta_{1}}^{1}, \ell_{\delta_{1}}^{2}, \ldots, \ell_{\delta_{1}}^{s}$ pairwise disjoint, it clearly follows that if $\rho-\sigma$ is sufficiently small, then $\psi$ is univalent on $\cup_{k=1}^{s} O_{\delta_{1}, \sigma}^{k}$. Thus, the claim is proven.

Now, fix $\delta \in(0, \rho)$ such that $\Sigma_{\delta, 1}, \Sigma_{\delta, 2}, \ldots, \Sigma_{\delta, s}$ are pairwise disjoint and $\psi$ has analytic continuations $\psi_{+}, \psi_{-}$to $\Sigma_{\delta, k}^{+}, \Sigma_{\delta, k}^{-}$, respectively, for each $1 \leq k \leq s$. By applying the claim above for an arbitrarily 
small value of $\delta^{\prime} \in(0, \delta)$, it clearly follows that for $\rho-\sigma$ sufficiently small, $\psi$ has an analytic continuation from $\mathbb{E}_{\rho}$ to $\operatorname{ext}\left(\Gamma_{\sigma}\right)$ with continuous boundary values on $\Gamma_{\sigma}$ when viewing each $\left[\sigma_{k}, \omega_{k}\right]$ as having two sides. Furthermore, every point $w_{0} \in \mathbb{T}_{\rho} \backslash\left\{\omega_{1}, \omega_{2}, \ldots, \omega_{s}\right\}$ has a neighborhood on which $\psi$ is one-to-one. This proves Lemma 3.1(a).

Suppose now that the statement of Lemma 3.1(b) is not true, that is, suppose there is a sequence $\left\{\sigma_{n}\right\}_{n \geq 1}$, with $\sigma_{n} \nearrow \rho$, such that either $\psi$ is not one-to-one on

$$
A_{\delta, \sigma_{n}}:=\overline{\mathbb{E}_{\sigma_{n}}} \backslash \cup_{k=1}^{s} \overline{\Sigma_{\delta, k}},
$$

or

$$
\psi\left(A_{\delta, \sigma_{n}}\right) \cap \overline{\psi\left(\operatorname{ext}\left(\Gamma_{\sigma_{n}}\right) \backslash A_{\delta, \sigma_{n}}\right)} \neq \emptyset .
$$

Then we can find two sequences of points $\left\{w_{n, 0}\right\}_{n \geq 1},\left\{w_{n, 1}\right\}_{n \geq 1}$, such that for each $n \geq 1$,

$$
w_{n, 0} \neq w_{n, 1}, \quad w_{n, 0} \in A_{\delta, \sigma_{n}}, \quad w_{n, 1} \in \overline{\mathbb{E}}_{\sigma_{n}}
$$

and either one of the following three equalities holds true:

$$
\psi\left(w_{n, 0}\right)=\psi\left(w_{n, 1}\right), \quad \psi\left(w_{n, 0}\right)=\psi_{+}\left(w_{n, 1}\right), \quad \psi\left(w_{n, 0}\right)=\psi_{-}\left(w_{n, 1}\right) .
$$

By extracting subsequences if necessary, we can assume that

$$
w_{n, 0} \rightarrow w_{0} \in \overline{\mathbb{E}}_{\rho} \backslash \cup_{k=1}^{s} \Sigma_{\delta, k}, \quad w_{n, 1} \rightarrow w_{1} \in \overline{\mathbb{E}}_{\rho},
$$

and, by (52), $\psi\left(w_{0}\right)=\psi\left(w_{1}\right)$. But, in view of Assumption A.1, this is only possible if $w_{1}=w_{0} \in \overline{\mathbb{E}}_{\rho} \backslash$ $\left\{\omega_{1}, \omega_{2}, \ldots, \omega_{s}\right\}$, contradicting the fact that $\psi$ is univalent in some neighborhood of $w_{0}$.

Lemma 3.2. Let $\delta>0$ be such that $\psi$ has analytic continuations $\psi_{ \pm}$to $\Sigma_{\delta, k}^{ \pm}$. Then, for every $\epsilon>0$ and $\sigma \in(\rho-\delta, \rho)$ such that

$$
\psi_{ \pm}\left(\left[\sigma_{k}, \omega_{k}\right]\right) \subset D_{\epsilon}\left(z_{k}\right) \subset G_{1}
$$

we have

$$
\begin{aligned}
& \frac{1}{2 \pi i} \int_{\sigma_{k}}^{\omega_{k}}\left(\frac{\varphi^{\prime}(z) t^{n}}{\varphi\left(\psi_{+}(t)\right)-\varphi(z)}-\frac{\varphi^{\prime}(z) t^{n}}{\varphi\left(\psi_{-}(t)\right)-\varphi(z)}\right) d t \\
= & -\left(\begin{array}{c}
n \\
-\lambda_{k}-1
\end{array}\right) \rho^{n+\lambda_{k}+1}\left(L\left(z_{k}, z\right) A_{k} e^{i\left(n+\lambda_{k}+1\right) \Theta_{k}}+r_{\sigma_{k}, n}(z)\right)
\end{aligned}
$$

where

$$
r_{\sigma_{k}, n}(z)= \begin{cases}\mathcal{O}\left(n^{-\lambda_{k}}\right), & \text { if } 0<\lambda_{k}<1, \lambda_{k} \neq 1 / 2, \\ \mathcal{O}\left(n^{-1} \log n\right), & \text { if } \lambda_{k}=1 / 2, \\ \mathcal{O}\left(n^{-1}\right), & \text { if } 1<\lambda_{k}<2\end{cases}
$$

uniformly on $G_{1} \backslash D_{\epsilon}\left(z_{k}\right)$ as $n \rightarrow \infty$.

Proof. For the analytic functions $\left(w-\omega_{k}\right)^{\beta}, \log \left(w-\omega_{k}\right)$, defined on $\Sigma_{\delta, k} \cap \mathbb{E}_{\rho}$ and corresponding to the branch of the argument

$$
\Theta_{k}-\pi<\arg \left(w-\omega_{k}\right)<\Theta_{k}+\pi, \quad w \in \mathbb{C} \backslash\left\{t \omega_{k}: t \leq 1\right\},
$$

let us denote by $\left(w-\omega_{k}\right)_{ \pm}^{\beta}$ and $\log _{ \pm}\left(w-\omega_{k}\right)$ their analytic continuations from $\Sigma_{\delta, k} \cap \mathbb{E}_{\rho}$ onto $\Sigma_{\delta, k}^{ \pm}$, respectively. For an integer $n \geq 0$ and real $\beta>-1$, we have (see, e.g., [12, Sec. 2])

$$
\begin{gathered}
\int_{0}^{1} x^{n}(1-x)^{\beta} \log (1-x) d x=-\frac{\Gamma(\beta+1) n ! \log n[1+\mathcal{O}(1 / \log n)]}{\Gamma(n+\beta+2)} \quad(n \rightarrow \infty), \\
\int_{0}^{1} x^{n}(1-x)^{\beta} d x=\frac{\Gamma(\beta+1) n !}{\Gamma(n+\beta+2)}=\frac{\Gamma(\beta+1)(1+o(1))}{n^{\beta+1}} \quad(n \rightarrow \infty),
\end{gathered}
$$


so that

$$
\begin{aligned}
& \int_{\sigma_{k}}^{\omega_{k}} t^{n}\left(t-\omega_{k}\right)_{ \pm}^{\beta} \log _{ \pm}\left(t-\omega_{k}\right) d t \\
= & e^{\mp i \beta \pi} \omega_{k}^{n+1+\beta} \int_{0}^{1} x^{n}(1-x)^{\beta}\left[\log (1-x)+\log \rho+i\left(\Theta_{k} \mp \pi\right)\right] d x+\mathcal{O}\left(\sigma^{n}\right) \\
= & -\frac{e^{\mp i \beta \pi} \omega_{k}^{n+1+\beta} \Gamma(\beta+1) n !(\log n)}{\Gamma(n+\beta+2)}+\mathcal{O}\left(\frac{\rho^{n}}{n^{\beta+1}}\right)+\mathcal{O}\left(\sigma^{n}\right) .
\end{aligned}
$$

From this last equality and the well-known identity

$$
\Gamma(1-z) \Gamma(z)=-\Gamma(-z) \Gamma(z+1)=\pi / \sin (\pi z),
$$

we then obtain

$$
\begin{aligned}
& \int_{\sigma_{k}}^{\omega_{k}} t^{n}\left[\left(t-\omega_{k}\right)_{+}^{\beta} \log _{+}\left(t-\omega_{k}\right)-\left(t-\omega_{k}\right)_{-}^{\beta} \log _{-}\left(t-\omega_{k}\right)\right] d t \\
= & -\frac{2 \pi i n !(\log n)\left(\omega_{k}\right)^{n+1+\beta}}{\Gamma(-\beta) \Gamma(n+\beta+2)}+\mathcal{O}\left(\frac{\rho^{n}}{n^{\beta+1}}\right)+\mathcal{O}\left(\sigma^{n}\right) \\
= & -2 \pi i\left(\begin{array}{c}
n \\
-\beta-1
\end{array}\right)(\log n)\left(\omega_{k}\right)^{n+1+\beta}+\mathcal{O}\left(\frac{\rho^{n}}{n^{\beta+1}}\right) .
\end{aligned}
$$

Similarly,

$$
\int_{\sigma_{k}}^{\omega_{k}} t^{n}\left[\left(t-\omega_{k}\right)_{+}^{\beta}-\left(t-\omega_{k}\right)_{-}^{\beta}\right] d t=2 \pi i\left(\begin{array}{c}
n \\
-\beta-1
\end{array}\right)\left(\omega_{k}\right)^{n+1+\beta}+\mathcal{O}\left(\sigma^{n}\right),
$$

and

$$
\int_{\sigma_{k}}^{\omega_{k}} \mathcal{O}\left(\left(t-\omega_{k}\right)_{ \pm}^{\beta} t^{n}\right) d t=\mathcal{O}\left(\frac{\rho^{n}}{n^{1+\beta}}\right) .
$$

Therefore, we get from (45), (53)-(55) that if $0<\lambda_{k}<1, \lambda_{k} \neq 1 / 2$, then

$$
\begin{aligned}
& \frac{1}{2 \pi i} \int_{\sigma_{k}}^{\omega_{k}}\left(\frac{\varphi^{\prime}(z) t^{n}}{\varphi\left(\psi_{+}(t)\right)-\varphi(z)}-\frac{\varphi^{\prime}(z) t^{n}}{\varphi\left(\psi_{-}(t)\right)-\varphi(z)}\right) d t \\
= & -\left(\begin{array}{c}
n \\
-\lambda_{k}-1
\end{array}\right) L\left(z_{k}, z\right) A_{k}\left(\omega_{k}\right)^{n+\lambda_{k}+1}-\left(\begin{array}{c}
n \\
-2 \lambda_{k}-1
\end{array}\right)\left[L\left(z_{k}, z\right) c_{020}^{k}+\frac{\partial L}{\partial \zeta}\left(z_{k}, z\right) \frac{\left(A_{k}\right)^{2}}{2}\right]\left(\omega_{k}\right)^{n+2 \lambda_{k}+1} \\
& +\left\{\begin{array}{cc}
\mathcal{O}\left(\rho^{n} / n^{2+\lambda_{k}}\right), & 1 / 2<\lambda_{k}<1, \\
\mathcal{O}\left(\rho^{n} / n^{3 \lambda_{k}+1}\right), & 0<\lambda_{k}<1 / 2,
\end{array}\right. \\
= & -\left(\begin{array}{c}
n \\
-\lambda_{k}-1
\end{array}\right)\left(\begin{array}{c}
L\left(z_{k}, z\right) A_{k}\left(\omega_{k}\right)^{n+\lambda_{k}+1}+\frac{\left[L\left(z_{k}, z\right) c_{020}^{k}+\frac{\partial L}{\partial \zeta}\left(z_{k}, z\right) \frac{\left(A_{k}\right)^{2}}{2}\right] \Gamma\left(-\lambda_{k}\right)\left(\omega_{k}\right)^{n+2 \lambda_{k}+1}}{\Gamma\left(-2 \lambda_{k}\right) n^{\lambda_{k}}} \\
\left.+o\left(\frac{\rho^{n}}{n^{\lambda_{k}}}\right)\right)
\end{array}\right.
\end{aligned}
$$

while if $\lambda_{k}=1 / 2$, then

$$
\begin{aligned}
& \frac{1}{2 \pi i} \int_{\sigma_{k}}^{\omega_{k}}\left(\frac{\varphi^{\prime}(z) t^{n}}{\varphi\left(\psi_{+}(t)\right)-\varphi(z)}-\frac{\varphi^{\prime}(z) t^{n}}{\varphi\left(\psi_{-}(t)\right)-\varphi(z)}\right) d t \\
= & -\left(\begin{array}{c}
n \\
-\lambda_{k}-1
\end{array}\right)\left(L\left(z_{k}, z\right) A_{k}\left(\omega_{k}\right)^{n+\lambda_{k}+1}-\frac{L\left(z_{k}, z\right) c_{111}^{k} \Gamma\left(-\lambda_{k}\right)\left(\omega_{k}\right)^{n+3 \lambda_{k}+1}(\log n)}{\Gamma\left(-3 \lambda_{k}\right) n^{2 \lambda_{k}}}+o\left(\frac{\rho^{n} \log n}{n^{2 \lambda_{k}}}\right)\right)(5
\end{aligned}
$$

Similarly, if $1<\lambda_{k}<2$, then we obtain from (46) and (54)-(55) that

$$
\begin{aligned}
& \frac{1}{2 \pi i} \int_{\sigma_{k}}^{\omega_{k}}\left(\frac{\varphi^{\prime}(z) t^{n}}{\varphi\left(\psi_{+}(t)\right)-\varphi(z)}-\frac{\varphi^{\prime}(z) t^{n}}{\varphi\left(\psi_{-}(t)\right)-\varphi(z)}\right) d t \\
= & -\left(\begin{array}{c}
n \\
-\lambda_{k}-1
\end{array}\right)\left(L\left(z_{k}, z\right) A_{k} \omega_{k}^{n+\lambda_{k}+1}+\frac{L\left(z_{k}, z\right) c_{110}^{k} \Gamma\left(-\lambda_{k}\right) \omega_{k}^{n+\lambda_{k}+2}}{\Gamma\left(-\lambda_{k}-1\right) n}+o\left(\frac{\rho^{n}}{n}\right)\right) .
\end{aligned}
$$


Lemma 3.2 is nothing but an abbreviation of relations (56), (57) and (58).

\subsection{Proof of Theorems 2.4 and 2.5 .}

Proof of Theorems 2.4. Let $\delta \in(0, \rho)$ be such that $\Sigma_{\delta, 1}, \Sigma_{\delta, 2}, \ldots, \Sigma_{\delta, s}$ are pairwise disjoint and $\psi$ has analytic continuations $\psi_{ \pm}$to $\Sigma_{\delta, k}^{ \pm}$for each $k \in\{1,2, \ldots, s\}$. Let $F \subset G_{\rho}$ be a compact set, so that by (14) we have that for any (fixed) $r \in(\rho, 1)$,

$$
P_{n}(z)=\frac{\sqrt{n+1}}{2 \pi i} \oint_{\mathbb{T}_{1}} \frac{\varphi^{\prime}(z) t^{n} d t}{\varphi(\psi(t))-\varphi(z)}+\mathcal{O}\left(\sqrt{n}(r \rho)^{n}\right), \quad z \in E .
$$

Now, it is clear that we can find $\epsilon>0$ and $\sigma \in(\rho-\delta, \rho)$ such that for every $k \in\{1,2, \ldots, s\}$,

$$
\psi_{ \pm}\left(\left[\sigma_{k}, \omega_{k}\right]\right) \subset D_{\epsilon}\left(z_{k}\right) \subset G_{1} \backslash F
$$

In addition, we can assume that $\sigma$ was chosen so close to $\rho$ that it satisfies the thesis of Lemma 3.1(a) and that

$$
\overline{\psi\left(\operatorname{ext}\left(\Gamma_{\sigma}\right) \cap \mathbb{D}_{1}\right)} \subset \bar{G}_{1} \backslash F .
$$

This last inclusion implies that for each $z \in F$, the function $[\varphi(\psi(w))-\varphi(z)]^{-1}$ is analytic on $\mathbb{D}_{1} \cap \operatorname{ext}\left(\Gamma_{\sigma}\right)$, with continuous boundary values on $\mathbb{T}_{1} \cup \Gamma_{\sigma}$ when viewing each $\left[\sigma_{k}, \omega_{k}\right]$ as having two sides. Hence, by deforming the path of integration in (59) from $\mathbb{T}_{1}$ to $\Gamma_{\sigma}$, and taking into account (60), Lemma 3.2 and (61), we obtain that for all $z \in F$,

$$
\begin{aligned}
P_{n}(z)= & \frac{\sqrt{n+1}}{2 \pi i} \oint_{\mathbb{T}_{\sigma}} \frac{\varphi^{\prime}(z) t^{n} d t}{\varphi(\psi(t))-\varphi(z)}+\mathcal{O}\left(\sqrt{n}(r \rho)^{n}\right) \\
& +\sqrt{n+1} \sum_{k=1}^{s} \frac{1}{2 \pi i} \int_{\sigma_{k}}^{\omega_{k}}\left(\frac{\varphi^{\prime}(z) t^{n}}{\varphi\left(\psi_{+}(t)\right)-\varphi(z)}-\frac{\varphi^{\prime}(z) t^{n}}{\varphi\left(\psi_{-}(t)\right)-\varphi(z)}\right) d t \\
= & \mathcal{O}\left(\sqrt{n}(r \rho)^{n}\right)+\mathcal{O}\left(\sqrt{n} \sigma^{n}\right)-\sqrt{n+1} \sum_{k=1}^{s}\left(\begin{array}{c}
n \\
-\lambda_{k}-1
\end{array}\right) \rho^{n+\lambda_{k}+1}\left(L\left(z_{k}, z\right) A_{k} e^{i\left(n+\lambda_{k}+1\right) \Theta_{k}}+r_{\sigma_{k}, n}(z)\right) \\
= & -\sqrt{n+1}\left(\begin{array}{c}
n \\
-\lambda_{1}-1
\end{array}\right) \rho^{n+\lambda_{1}+1}\left(\sum_{k=1}^{u} L\left(z_{k}, z\right) A_{k} e^{i\left(n+\lambda_{k}+1\right) \Theta_{k}}+R_{n}(z)\right)
\end{aligned}
$$

where

$$
R_{n}(z)=\left\{\begin{array}{ll}
\mathcal{O}\left(n^{-\lambda_{1}}\right), & \text { if } 0<\lambda_{1}<1, \lambda_{1} \neq 1 / 2, \\
\mathcal{O}\left(n^{-1} \log n\right), & \text { if } \lambda_{1}=1 / 2, \\
\mathcal{O}\left(n^{-1}\right), & \text { if } 1<\lambda_{1}<2,
\end{array}+ \begin{cases}\mathcal{O}\left(n^{\lambda_{1}-\lambda_{u+1}}\right), & \text { if } u<s, \\
0, & \text { if } u=s\end{cases}\right.
$$

uniformly on $F$ as $n \rightarrow \infty$. This proves Theorem 2.4 .

Proof of Theorems 2.5. Let $\delta \in(0, \rho)$ be such that $\Sigma_{\delta, 1}, \Sigma_{\delta, 2}, \ldots, \Sigma_{\delta, s}$ are pairwise disjoint and $\psi$ has analytic continuations $\psi_{ \pm}$to $\Sigma_{\delta, k}^{ \pm}$for each $k \in\{1,2, \ldots, s\}$. Since, by Assumption A.1, $\psi\left(\overline{\mathbb{E}}_{\rho} \backslash \cup_{k=1}^{s} \Sigma_{\delta, k}\right)$ is a closed set that does not contain any corner $z_{k}$, we can find $\epsilon>0$ and $\sigma \in(\rho-\delta, \rho)$ such that for every $k \in\{1,2, \ldots, s\}$,

$$
\psi_{ \pm}\left(\left[\sigma_{k}, \omega_{k}\right]\right) \subset D_{\epsilon}\left(z_{k}\right) \subset G_{1}, \quad D_{\epsilon}\left(z_{k}\right) \cap \psi\left(\mathbb{E}_{\sigma} \backslash \cup_{k=1}^{s} \overline{\Sigma_{\delta, k}}\right)=\emptyset .
$$

In addition, of course, we can assume that $\sigma$ satisfies the thesis of Lemma 3.1, so that for each $z \in V_{\delta, \sigma}:=$ $\psi\left(\mathbb{E}_{\sigma} \backslash \cup_{k=1}^{s} \overline{\Sigma_{\delta, k}}\right)$, the function $[\varphi(\psi(w))-\varphi(z)]^{-1}$ is analytic on $\left[\mathbb{D}_{1} \cap \operatorname{ext}\left(\Gamma_{\sigma}\right)\right] \backslash\{\phi(z)\}$, with continuous boundary values on $\mathbb{T}_{1} \cup \Gamma_{\sigma}$ and a simple pole at $\phi(z)$. Then, we get from (14) and the residue theorem that

$$
\begin{aligned}
P_{n}(z)= & \frac{\sqrt{n+1}}{2 \pi i} \oint_{\mathbb{T}_{1}} \frac{\varphi^{\prime}(z) t^{n} d t}{\varphi(\psi(t))-\varphi(z)}+\epsilon_{n}(z) \\
= & \sqrt{n+1} \phi^{\prime}(z)[\phi(z)]^{n}+\frac{\sqrt{n+1}}{2 \pi i} \oint_{\mathbb{T}_{\sigma}} \frac{\varphi^{\prime}(z) t^{n} d t}{\varphi(\psi(t))-\varphi(z)}+\epsilon_{n}(z) \\
& +\sqrt{n+1} \sum_{k=1}^{s} \frac{1}{2 \pi i} \int_{\sigma_{k}}^{\omega_{k}}\left(\frac{\varphi^{\prime}(z) t^{n}}{\varphi\left(\psi_{+}(t)\right)-\varphi(z)}-\frac{\varphi^{\prime}(z) t^{n}}{\varphi\left(\psi_{-}(t)\right)-\varphi(z)}\right) d t, \quad z \in V_{\delta, \sigma} .
\end{aligned}
$$


Let then $E \subset V_{\delta, \sigma}$ be a compact set and let $r \in(\rho, 1)$ be such that $E \subset \bar{G}_{r}$, so that $\epsilon_{n}(z)=\mathcal{O}\left(\sqrt{n}(r \rho)^{n}\right)$ uniformly on $E$ as $n \rightarrow \infty$. On the other hand, again by Lemma 3.1, the set

$$
\psi\left(\Gamma_{\sigma}\right):=\overline{\psi\left(\operatorname{ext}\left(\Gamma_{\sigma}\right)\right)} \backslash \psi\left(\operatorname{ext}\left(\Gamma_{\sigma}\right)\right)
$$

is compact and disjoint from $E$, so that

$$
\min \left\{|\varphi(\zeta)-\varphi(z)|: \zeta \in \psi\left(\Gamma_{\sigma}\right), z \in E\right\}>0
$$

Therefore, we get from (64) by considering (63), Lemma 3.2 and (65) that for all $z \in E$,

$$
\begin{aligned}
P_{n}(z)= & \sqrt{n+1} \phi^{\prime}(z)[\phi(z)]^{n}+\mathcal{O}\left(\sqrt{n} \sigma^{n}\right)+\mathcal{O}\left(\sqrt{n}(r \rho)^{n}\right) \\
& -\sqrt{n+1} \sum_{k=1}^{s}\left(\begin{array}{c}
n \\
-\lambda_{k}-1
\end{array}\right) \rho^{n+\lambda_{k}+1}\left(L\left(z_{k}, z\right) A_{k} e^{i\left(n+\lambda_{k}+1\right) \Theta_{k}}+r_{\sigma_{k}, n}(z)\right) \\
= & \sqrt{n+1} \phi^{\prime}(z)[\phi(z)]^{n}-\sqrt{n+1}\left(\begin{array}{c}
n \\
-\lambda_{1}-1
\end{array}\right) \rho^{n+\lambda_{1}+1}\left(\sum_{k=1}^{u} L\left(z_{k}, z\right) A_{k} e^{i\left(n+\lambda_{k}+1\right) \Theta_{k}}+R_{n}(z)\right),
\end{aligned}
$$

where $R_{n}(z)$ satisfies (62) uniformly on $E$ as $n \rightarrow \infty$.

Thus, it only remains to prove Theorem 2.5(b). Let $\delta^{\prime}>0$ and $\epsilon>0$ be such that $\Sigma_{\delta^{\prime}, 1}, \Sigma_{\delta^{\prime}, 2}, \ldots, \Sigma_{\delta^{\prime}, s}$ are pairwise disjoint, $\psi$ has analytic continuations $\psi_{ \pm}$to $\Sigma_{\delta^{\prime}, k}^{ \pm}$for each $k \in\{1,2, \ldots, s\}$,

$$
\overline{\psi_{ \pm}\left(\Sigma_{\delta^{\prime}, k}^{ \pm}\right)} \subset D_{\epsilon}\left(z_{k}\right) \subset G_{1}, \quad 1 \leq k \leq s
$$

and

$$
z_{k} \neq z_{j} \Rightarrow D_{\epsilon}\left(z_{k}\right) \cap D_{\epsilon}\left(z_{j}\right)=\emptyset
$$

Since for every $1 \leq k \leq s$,

$$
\lim _{w \rightarrow \omega_{k}} \frac{\psi_{ \pm}(w)-z_{k}}{\left(w-\omega_{k}\right)^{\lambda_{k}}}=A_{k}>0
$$

we can assume $\delta^{\prime}$ was chosen so small that

$$
z_{k} \notin \psi_{ \pm}\left(\Sigma_{\delta^{\prime}, k}^{ \pm}\right), \quad 1 \leq k \leq s
$$

Then, fix $\delta \in\left(0, \delta^{\prime}\right)$, and for this $\delta$, choose $\sigma \in(0, \rho)$ satisfying Lemma 3.1. Let $z_{j}$ be a (fixed) corner of $L_{\rho}$, and for each $k$ such that $\psi\left(\omega_{k}\right)=z_{j}$, let $\ell_{k} \subset \Sigma_{\delta, k} \cap \overline{\mathbb{E}}_{\rho}$ be a positively oriented closed simple path encircling the segment $\left(\sigma_{k}, \omega_{k}\right]$, whose only common point with $\Gamma_{\sigma}$ is $\sigma_{k}$.

By Lemma 3.1, (66), (67) and (68), the function $\left[\varphi(\psi(w))-\varphi\left(z_{j}\right)\right]^{-1}$ is analytic on $\mathbb{D}_{1} \cap \operatorname{ext}\left(\Gamma_{\sigma}\right)$, with continuous boundary values on $\mathbb{T}_{1} \cup \Gamma_{\sigma} \backslash\left\{\omega_{k}: \psi\left(\omega_{k}\right)=z_{j}\right\}$. Hence, we obtain from (14) that (with $\rho<r<1$ )

$$
\begin{aligned}
P_{n}\left(z_{j}\right)= & \sum_{k: z_{k} \neq z_{j}} \frac{\sqrt{n+1}}{2 \pi i} \int_{\sigma_{k}}^{\omega_{k}}\left(\frac{\varphi^{\prime}\left(z_{j}\right) t^{n}}{\varphi\left(\psi_{+}(t)\right)-\varphi\left(z_{j}\right)}-\frac{\varphi^{\prime}\left(z_{j}\right) t^{n}}{\varphi\left(\psi_{-}(t)\right)-\varphi\left(z_{j}\right)}\right) d t \\
& +\sum_{k: z_{k}=z_{j}} \frac{\sqrt{n+1}}{2 \pi i} \oint_{\ell_{k}} \frac{\varphi^{\prime}\left(z_{j}\right) t^{n} d t}{\varphi(\psi(t))-\varphi\left(z_{j}\right)}+\mathcal{O}\left(\sqrt{n} \sigma^{n}\right)+\mathcal{O}\left(\sqrt{n}(r \rho)^{n}\right) .
\end{aligned}
$$


Now, after integrating by parts a couple of times over $\ell_{k}$ and using (54) we get

$$
\begin{aligned}
\oint_{\ell_{k}} t^{n}\left(t-\omega_{k}\right)^{-\lambda_{k}} d t= & \frac{\left(\sigma_{k}\right)^{n}\left[\left(\sigma_{k}-\omega_{k}\right)_{-}^{1-\lambda_{k}}-\left(\sigma_{k}-\omega_{k}\right)_{+}^{1-\lambda_{k}}\right]}{\left(1-\lambda_{k}\right)} \\
& -\frac{n\left(\sigma_{k}\right)^{n-1}\left[\left(\sigma_{k}-\omega_{k}\right)_{-}^{2-\lambda_{k}}-\left(\sigma_{k}-\omega_{k}\right)_{+}^{2-\lambda_{k}}\right]}{\left(1-\lambda_{k}\right)\left(2-\lambda_{k}\right)} \\
& +\frac{n(n-1)}{\left(1-\lambda_{k}\right)\left(2-\lambda_{k}\right)} \oint_{\ell_{k}} t^{n-2}\left(t-\omega_{k}\right)^{2-\lambda_{k}} d t \\
= & \frac{n(n-1)}{\left(1-\lambda_{k}\right)\left(2-\lambda_{k}\right)} \int_{\sigma_{k}}^{\omega_{k}} t^{n-2}\left[\left(t-\omega_{k}\right)_{+}^{2-\lambda_{k}}-\left(t-\omega_{k}\right)_{-}^{2-\lambda_{k}}\right] d t+\mathcal{O}\left(n \sigma^{n}\right) \\
= & \frac{2 \pi i n !\left(\omega_{k}\right)^{n-\lambda_{k}+1}}{\Gamma\left(\lambda_{k}\right) \Gamma\left(n+2-\lambda_{k}\right)}+\mathcal{O}\left(n^{2} \sigma^{n}\right) .
\end{aligned}
$$

Then, combining (69), Lemma 3.2, relations (48)-(151) and (70), we conclude that

$$
\begin{aligned}
\frac{P_{n}\left(z_{j}\right)}{\sqrt{n+1}}= & -\sum_{k: z_{k} \neq z_{j}}\left(\begin{array}{c}
n \\
-\lambda_{k}-1
\end{array}\right) \rho^{n+\lambda_{k}+1}\left(L\left(z_{k}, z_{j}\right) A_{k} e^{i\left(n+\lambda_{k}+1\right) \Theta_{k}}+o(1)\right) \\
& +\sum_{k: z_{k}=z_{j}}\left[\left(\begin{array}{c}
n \\
\lambda_{k}-1
\end{array}\right)\left(A_{k}\right)^{-1}\left(\omega_{k}\right)^{n-\lambda_{k}+1}+\left\{\begin{array}{ll}
\mathcal{O}\left(\rho^{n} / n^{1+\lambda_{k}}\right), & 0<\lambda_{k} \leq 1 / 2, \\
\mathcal{O}\left(\rho^{n} / n^{2-\lambda_{k}}\right), & 1 / 2<\lambda_{k}<2, \quad \lambda_{k} \neq 1,
\end{array}\right]\right. \\
& +\mathcal{O}\left(\sigma^{n}\right)+\mathcal{O}\left((r \rho)^{n}\right),
\end{aligned}
$$

so that if $\lambda_{j}^{*}=\max \left\{\lambda_{k}: z_{k}=z_{j}, 1 \leq k \leq s\right\}$, then

$$
\begin{aligned}
\frac{P_{n}\left(z_{j}\right)}{\sqrt{n+1}\left(\begin{array}{c}
n \\
\lambda_{j}^{*}-1
\end{array}\right)}-\sum_{\substack{k: z_{k}=z_{j} \\
\lambda_{k}=\lambda_{j}^{*}}}\left(A_{k}\right)^{-1}\left(\omega_{k}\right)^{n-\lambda_{j}^{*}+1}= & \begin{cases}\mathcal{O}\left(\rho^{n} / n^{2 \lambda_{j}^{*}}\right), & 0<\lambda_{j}^{*} \leq 1 / 2, \\
\mathcal{O}\left(\rho^{n} / n\right), & 1 / 2<\lambda_{j}^{*}<2, \lambda_{j}^{*} \neq 1,\end{cases} \\
& +\sum_{\substack{k: z_{k}=z_{j} \\
\lambda_{k}<\lambda_{j}^{*}}} \mathcal{O}\left(\frac{\rho^{n}}{n^{\lambda_{j}^{*}-\lambda_{k}}}\right)+\sum_{k: z_{k} \neq z_{j}} \mathcal{O}\left(\frac{\rho^{n}}{n^{\lambda_{k}+\lambda_{j}^{*}}}\right),
\end{aligned}
$$

that is Theorem 2.5(b) holds true.

3.5. Proof of Theorem 2.13. Proof of part (a): Fix $m \geq 0$ an integer, and let us show that $z^{s-1}\left(z^{s}-1\right)^{m}$ is orthogonal over $G_{1}$ to all powers $z^{n}$ with $0 \leq n \leq s m+s-2$ an integer. For this, first notice that since both $G_{1}$ and the area measure are invariant under a rotation of angle $e^{2 \pi i / s}$, we have that for any two integers $\alpha, \beta$ such that $\alpha-\beta \neq 0 \bmod s$,

$$
\int_{G_{1}} z^{\alpha} \overline{z^{\beta}} d A(z)=0
$$

and therefore, $z^{s-1}\left(z^{s}-1\right)^{m}=\sum_{j=0}^{m}\left(\begin{array}{c}m \\ j\end{array}\right)(-1)^{m-j} z^{s j+s-1}$ is orthogonal over $G_{1}$ to all powers $z^{s m^{\prime}+l}$ with $0 \leq m^{\prime} \leq m, 0 \leq l \leq s-2$ integers.

For the remaining powers, we obtain by applying Green's formula ([4, p. 10]) and making the change of variables $z=\left(R^{s} w^{s}+1\right)^{1 / s}$ that for $0 \leq m^{\prime} \leq m$,

$$
\begin{aligned}
\frac{1}{\pi} \int_{G_{1}} z^{s-1}\left(z^{s}-1\right)^{m} \overline{z^{s m^{\prime}+s-1}} d A(z) & =\frac{1}{s\left(m^{\prime}+1\right) 2 \pi i} \oint_{L_{1}} z^{s-1}\left(z^{s}-1\right)^{m} \overline{z^{s\left(m^{\prime}+1\right)}} d z \\
& =\frac{R^{s(m+1)}}{s\left(m^{\prime}+1\right) 2 \pi i} \oint_{\mathbb{T}_{1}} w^{s m+s-1} \overline{\left(R^{s} w^{s}+1\right)^{m^{\prime}+1}} d w \\
& = \begin{cases}0, & 0 \leq m^{\prime} \leq m-1, \\
R^{2 s(m+1)} /(s m+s), & m^{\prime}=m\end{cases}
\end{aligned}
$$


Proof of part (b): We have that $\rho=R^{-1}$, so that as shown in the proof of Theorem 2.4, if $F \subset G_{\rho}$ is compact, then for $r \in\left(R^{-1}, 1\right)$ and certain $\sigma \in\left(0, R^{-1}\right)$ sufficiently close to $R^{-1}$, we have that uniformly in $z \in F$ as $n \rightarrow \infty$,

$$
P_{n}(z)=\sum_{k=1}^{s} \frac{\sqrt{n+1}}{2 \pi i} \int_{\sigma_{k}}^{\omega_{k}}\left(\frac{\varphi^{\prime}(z) t^{n}}{\varphi\left(\psi_{+}(t)\right)-\varphi(z)}-\frac{\varphi^{\prime}(z) t^{n}}{\varphi\left(\psi_{-}(t)\right)-\varphi(z)}\right) d t+\mathcal{O}\left(\sqrt{n}(r / R)^{n}\right)+\mathcal{O}\left(\sqrt{n} \sigma^{n}\right) .
$$

Now, suppose $n=s m+l$, with $0 \leq l \leq s-2, m \geq 0$ integers. From (44) for $N=3 s-l-2$, we get that uniformly in $w \in \cup_{k=1}^{s}\left[\sigma_{k}, \omega_{k}\right], z \in F$,

$$
\frac{\varphi^{\prime}(z)}{\varphi\left(\psi_{ \pm}(w)\right)-\varphi(z)}=\frac{\varphi^{\prime}(z)}{\varphi(0)-\varphi(z)}-\sum_{j=0}^{3 s-l-3} \frac{\partial^{j} L}{\partial \zeta^{j}}(0, z) \frac{\left[\psi_{ \pm}(w)\right]^{j+1}}{(j+1) !}+\mathcal{O}\left(\left[\psi_{ \pm}(w)\right]^{3 s-l-1}\right)
$$

and since $e^{2 \pi i k / s} \psi(w)=\psi\left(e^{2 \pi i k / s} w\right)$ for all $1 \leq k \leq s$, we have in virtue of (172), (41), (42) and (55) that

$$
\begin{aligned}
P_{n}(z)= & -\sum_{j=0}^{3 s-l-3} \frac{\partial^{j} L}{\partial \zeta^{j}}(0, z) \frac{\left(\sum_{k=1}^{s} e^{2(k-1)(n+j+2) i \pi / s}\right) \sqrt{n+1}}{(j+1) ! 2 \pi i} \int_{\sigma_{1}}^{\omega_{1}} t^{n}\left(\left[\psi_{+}(t)\right]^{j+1}-\left[\psi_{-}(t)\right]^{j+1}\right) d t \\
& +\mathcal{O}\left(\sqrt{n} R^{-n} n^{-(4 s-l-1) / s}\right) \\
= & -\frac{\partial^{s-l-2} L}{\partial \zeta^{s-l-2}}(0, z) \frac{s \sqrt{n+1}}{(s-l-1) ! 2 \pi i} \int_{\sigma_{1}}^{\omega_{1}} t^{n}\left(\left[\psi_{+}(t)\right]^{s-l-1}-\left[\psi_{-}(t)\right]^{s-l-1}\right) d t \\
& -\frac{\partial^{2 s-l-2} L}{\partial \zeta^{2 s-l-2}}(0, z) \frac{s \sqrt{n+1}}{(2 s-l-1) ! 2 \pi i} \int_{\sigma_{1}}^{\omega_{1}} t^{n}\left(\left[\psi_{+}(t)\right]^{2 s-l-1}-\left[\psi_{-}(t)\right]^{2 s-l-1}\right) d t \\
& +\mathcal{O}\left(\sqrt{n} R^{-n} n^{-(4 s-l-1) / s}\right) .
\end{aligned}
$$

Now,

$$
\psi(w)=\left(R^{s} w^{s}+1\right)^{1 / s}=(R s)^{1 / s} e^{i \pi(s-1) / s^{2}}\left(w-\omega_{1}\right)^{1 / s}\left[1+\frac{(s-1) R e^{-i \pi / s}\left(w-\omega_{1}\right)}{2 s}+\mathcal{O}\left(\left(w-\omega_{1}\right)^{2}\right)\right],
$$

and therefore,

$$
\begin{aligned}
{[\psi(w)]^{s-l-1}=} & (R s)^{(s-l-1) / s} e^{i \pi(s-1)(s-l-1) / s^{2}}\left(w-\omega_{1}\right)^{(s-l-1) / s} \\
& \times\left[1+\frac{(s-1)(s-l-1) R e^{-i \pi / s}\left(w-\omega_{1}\right)}{2 s}+\mathcal{O}\left(\left(w-\omega_{1}\right)^{2}\right)\right]
\end{aligned}
$$

and

$$
[\psi(w)]^{2 s-l-1}=(R s)^{(2 s-l-1) / s} e^{i \pi(s-1)(2 s-l-1) / s^{2}}\left(w-\omega_{1}\right)^{(2 s-l-1) / s}\left[1+\mathcal{O}\left(w-\omega_{1}\right)\right] .
$$

Then, from (74) and (75), we obtain

$$
\begin{aligned}
\frac{1}{2 \pi i} \int_{\sigma_{1}}^{\omega_{1}} t^{n}\left(\left[\psi_{+}(t)\right]^{s-l-1}-\left[\psi_{-}(t)\right]^{s-l-1}\right) d t= & \frac{(-1)^{m} R^{-(n+1)} s^{(s-l-1) / s} n !}{\Gamma(n+(3 s-l-1) / s) \Gamma((1+l-s) / s)} \\
& +\frac{(-1)^{m} R^{-(n+1)} s^{-(l+1) / s}(s-1)(s-l-1) n !}{2 \Gamma(n+(4 s-l-1) / s) \Gamma((1+l-2 s) / s)} \\
& +\mathcal{O}\left(R^{-n} n^{-(4 s-l-1) / s}\right), \\
\frac{1}{2 \pi i} \int_{\sigma_{1}}^{\omega_{1}} t^{n}\left(\left[\psi_{+}(t)\right]^{2 s-l-1}-\left[\psi_{-}(t)\right]^{2 s-l-1}\right) d t= & \frac{(-1)^{m+1} R^{-(n+1)} s^{(2 s-l-1) / s} n !}{\Gamma(n+(4 s-l-1) / s) \Gamma((1+l-2 s) / s)} \\
& +\mathcal{O}\left(R^{-n} n^{-(4 s-l-1) / s}\right) .
\end{aligned}
$$

Theorem 2.13(b) follows immediately after inserting these two previous equalities into (73).

\subsection{Proofs of the zero results.}


Proof of Theorem 2.8. Suppose that for some subsequence $\left\{n_{\nu}\right\}_{\nu \geq 1} \subset \mathbb{N}$,

$$
H_{n_{\nu}}(z)=\varphi^{\prime}(z) \sum_{k=1}^{u} \frac{\varphi^{\prime}\left(z_{k}\right) \hat{A}_{k} e^{2 \pi i n_{\nu} \theta_{k}}}{\left[\varphi(z)-\varphi\left(z_{k}\right)\right]^{2}} \rightarrow f(z) \quad \text { as } \quad \nu \rightarrow \infty .
$$

By extracting a subsequence if necessary, we may assume that for some fixed $\ell \in\{1, \ldots, \mathbf{q}\}, n_{\nu}=\mathbf{q} m_{\nu}+\ell$ with $m_{\nu} \in \mathbb{N}$, and by the compacity of $\mathbb{T}_{1}$, that for some real numbers $\alpha_{2}, \ldots, \alpha_{u^{*}}$

$$
\lim _{\nu \rightarrow \infty} e^{2 \pi i r_{k j} n_{\nu} \theta_{j}}=e^{2 \pi i r_{k j} \alpha_{j}}, \quad 1 \leq k \leq u, 2 \leq j \leq u^{*},
$$

so that by (24), $f$ must have the form (25).

Conversely, we now show that given an integer $\ell \in\{1, \ldots, \mathbf{q}\}$ and arbitrary real numbers $\alpha_{2}, \ldots, \alpha_{u^{*}}$, it is possible to choose a subsequence $\left\{n_{\nu}\right\}_{\nu \geq 1}$ such that

$$
\lim _{\nu \rightarrow \infty} e^{2 \pi i n_{\nu} \theta_{k}}=\lim _{\nu \rightarrow \infty} e^{2 \pi i\left(\frac{n_{\nu} p_{k}}{q_{k}}+\sum_{j=2}^{u^{*}} r_{k j} n_{\nu} \theta_{j}\right)}=e^{2 \pi i\left(\frac{\ell_{p_{k}}}{q_{k}}+\sum_{j=2}^{u^{*}} r_{k j} \alpha_{j}\right)}, \quad 1 \leq k \leq u .
$$

For this, we first observe that given arbitrary real numbers $\chi_{2}, \ldots, \chi_{u}$, it is always possible to find a subsequence $\left\{m_{\nu}\right\}_{\nu \geq 1} \subset \mathbb{N}$ such that

$$
\lim _{\nu \rightarrow \infty} e^{2 \pi i r_{k j} \mathbf{q} m_{\nu} \theta_{j}}=e^{2 \pi i r_{k j} \mathbf{q} \chi_{j}}, \quad 1 \leq k \leq u, 2 \leq j \leq u^{*} .
$$

In effect, consider the set of linear forms in the variable $x$

$$
\left\{r_{k j} \mathbf{q} \theta_{j} x: 1 \leq k \leq u, 2 \leq j \leq u^{*}\right\},
$$

and suppose $\beta_{k j}, 1 \leq k \leq u, 2 \leq j \leq u^{*}$, are integers such that

$$
\sum_{k, j} \beta_{k j} r_{k j} \mathbf{q} \theta_{j} x=x \sum_{j=2}^{u^{*}}\left(\sum_{k=1}^{u} \beta_{k j} r_{k j} \mathbf{q}\right) \theta_{j}
$$

is a linear form whose coefficient is an integer. Then, by the linear independence of the numbers $1, \theta_{2}, \ldots \theta_{u^{*}}$, we must have $\sum_{k=1}^{u} \beta_{k j} r_{k j} \mathbf{q}=0$ for every $2 \leq j \leq u^{*}$. Hence, for an arbitrary collection of real numbers $\chi_{2}, \ldots, \chi_{u^{*}}$, we have $\sum_{k, j} \beta_{k j} r_{k j} \mathbf{q} \theta_{j} \chi_{j}=0$, and so by Kronecker's theorem [2, Chap. III, Thm. IV.], it is possible to find a subsequence $\left\{m_{\nu}\right\}_{\nu \geq 1}$ satisfying (77).

Then, choose a subsequence $\left\{m_{\nu}\right\}_{\nu \geq 1}$ satisfying (77) with $\chi_{j}=\left(\alpha_{j}-\ell \theta_{j}\right) / \mathbf{q}, 2 \leq j \leq u^{*}$. Then, (76) is satisfied by the subsequence $n_{\nu}:=\mathbf{q} m_{\nu}+\ell, \nu \in \mathbb{N}$.

It only remains to prove that there is a function $f$ of the form (25) that is not identically zero. Assume without loss of generality that the set $\left\{k: z_{k}=z_{1}, 1 \leq k \leq u\right\}$ consists of the numbers $1,2, \ldots, u^{\prime}$ for some $u^{\prime} \leq u$. It suffices to show that it is impossible to have

$$
\sum_{k=1}^{u^{\prime}} \varphi^{\prime}\left(z_{k}\right) \hat{A}_{k} e^{2 \pi i\left(\frac{\ell p_{k}}{q_{k}}+\sum_{j=2}^{u^{*}} r_{k j} \alpha_{j}\right)}=0, \quad \ell \in\{1, \ldots, \mathbf{q}\}, \alpha_{2}, \ldots, \alpha_{u^{*}} \in \mathbb{R} .
$$

Assume, on the contrary, that this is the case. Since $\varphi^{\prime}\left(z_{1}\right) \hat{A}_{1} \neq 0$ and $r_{1 j}=0$ for all $2 \leq j \leq u^{*}$, we must obviously have $r_{k j}=0$ for all $1 \leq k \leq u^{\prime}, 2 \leq j \leq u^{*}$, and consequently,

$$
\theta_{k}=\frac{p_{k}}{q_{k}}, \quad k=1,2, \ldots, u^{\prime} .
$$

Let $q^{\prime} \leq \mathbf{q}$ be the least common multiple of the denominators $q_{1}, q_{2}, \ldots, q_{u^{\prime}}$, and for $k=1,2, \ldots, u^{\prime}$, set $p_{k}^{\prime}:=p_{k} q^{\prime} / q_{k}$, so that $1 \leq p_{k}^{\prime} \leq q^{\prime}$, and since $\theta_{1}, \theta_{2}, \ldots, \theta_{u^{\prime}}$ are pairwise distinct, so are the numbers $p_{1}^{\prime}, p_{2}^{\prime}, \ldots, p_{u^{\prime}}^{\prime}$, and therefore $u^{\prime} \leq q^{\prime}$. Then, by (78) we must have

$$
\sum_{k=1}^{u^{\prime}} \varphi^{\prime}\left(z_{k}\right) \hat{A}_{k}\left(e^{2 \pi i \ell / q^{\prime}}\right)^{p_{k}^{\prime}}=0 \quad \forall \ell \in\left\{1, \ldots, q^{\prime}\right\} .
$$

But this homogenous system of linear equations with unknowns $\varphi^{\prime}\left(z_{k}\right) \hat{A}_{k}, 1 \leq k \leq u^{\prime}$, has only the trivial solution, since the Vandermonde matrix $\left(a_{l, m}\right)_{1 \leq \ell, m \leq q^{\prime}}, a_{l, m}=\left(e^{2 \pi i \ell / q^{\prime}}\right)^{m}$, is nonsingular. This contradicts that all the numbers $\varphi^{\prime}\left(z_{k}\right) \hat{A}_{k}$ are nonzero. 
Proof of Corollary 2.10. Part (a): This is just a straightforward consequence of Theorem 2.8 and Hurwitz's theorem, therefore, we omit it.

Part (b): Suppose there is a compact set $E \subset G_{\rho}$ and a subsequence $\left\{n_{j}\right\} \subset \mathbb{N}$ such that $P_{n_{j}}^{*}$ has more than 2(J-1) zeros on $E$ counting multiplicities, where $J$ is the number of corners of $L_{\rho}(J \leq s)$. By Assumption A.3 (and extracting a subsequence from $\left\{n_{j}\right\}$ if needed), we can assume that $\left\{P_{n_{j}}^{*}\right\}$ converges locally uniformly on $G_{\rho}$ to a nonzero function of the form $R(\varphi(z))$, where $R(w)$ is a rational function with numerator having degree no larger than $2(J-1)$. By Hurwitz's theorem, there is an open set $U \supset E$ such that for all $j$ large enough, $P_{n_{j}}^{*}$ and $R(\varphi(z))$ have the same number of zeros on $U$, contradicting our assumption.

We now show that $\nu_{n} \stackrel{*}{\longrightarrow} \mu_{L_{\rho}}$, for which we use standard arguments. By Helly's selection theorem [15, Thm. 1.3], from every subsequence of $\left\{\nu_{n}\right\}_{n \geq 1}$ it is possible to extract another subsequence converging in the weak*-topology to a measure $\mu$. Thus, to finish the proof, it suffices to show that every such limit measure $\mu$ is the equilibrium measure $\mu_{L_{\rho}}$ of $L_{\rho}$.

Then, suppose $\nu_{n_{j}} \stackrel{*}{\longrightarrow} \mu$ as $j \rightarrow \infty$, so that by Corollary 2.7 and what we just proved above, $\mu$ must be supported on $L_{\rho}$. Let us denote by $U^{\alpha}(z)$ the logarithmic potential of the measure $\alpha$, that is,

$$
U^{\alpha}(z):=\int \log \frac{1}{|z-t|} d \alpha(t) .
$$

Then, we obtain from Theorem 2.1 and the fact that $\nu_{n_{j}} \stackrel{*}{\longrightarrow} \mu$ that for all $z \in \Omega_{\rho}$

$$
U^{\mu}(z)=\lim _{j \rightarrow \infty} U^{\nu_{n_{j}}}(z)=\lim _{j \rightarrow \infty} \frac{1}{n_{j}} \log \frac{\kappa_{n_{j}}}{\left|P_{n_{j}}(z)\right|}=\log \left|\phi^{\prime}(\infty) / \phi(z)\right| .
$$

On the other hand, it is not difficult to see from the definition of $\mu_{L_{\rho}}$ in (20) that for all $z \in \Omega_{\rho}, U^{\mu_{L_{\rho}}}(z)=$ $\log \left|\phi^{\prime}(\infty) / \phi(z)\right|$. Hence, $\mu$ and $\mu_{L_{\rho}}$ are two measures supported on $L_{\rho}$ whose logarithmic potentials coincide in $\Omega_{\rho}$, which in view of Carleson's theorem [15, Thm. 4.13] forces $\mu=\mu_{L_{\rho}}$.

Proof of Corollary 2.9. By Theorem 2.8, there is a subsequence $\left\{n_{j}\right\} \subset \mathbb{N}$ such that $\left\{P_{n_{j}}^{*}\right\}$ converges locally uniformly on $G_{\rho}$ to a nonzero function of the form (25). Then, proceeding exactly as in the proof of Corollary

2.10(b), we find that $\nu_{n_{j}} \stackrel{*}{\longrightarrow} \mu_{L_{\rho}}$ as $j \rightarrow \infty$, and since the support of the equilibrium measure $\mu_{L_{\rho}}$ is the whole of $L_{\rho}$, we must have $L_{\rho} \subset \mathcal{Z}$.

\section{REFERENCES}

[1] Carleman, T. 1922. Über die approximation analytischer funktionen durch lineare aggregate von vorgegebenen potenzen. Archiv. för Math. Atron. och Fysik 17: 1-30.

[2] Cassels, J. W. S. 1957. An introduction to diophantine approximation. Cambridge Tracts in Mathematics and Mathematical Physics. New York: Cambridge University Press.

[3] Davis, P. J. 1974. The Schwarz function and its applications. The Carus Mathematical Monographs 17. The Mathematical Association of America.

[4] Gaier, D. 1987. Lectures on complex approximation. Boston: Birkhäuser.

[5] Knopp, K. 1952. Theory of functions, Vol. 2. New York: Dover.

[6] Korovkin, P. P. 1947. The asymptotic representation of polynomials orthogonal over a region. Dokl. Akad. Nauk. SSSR. 58: 1883-1885 (in Russian).

[7] Lehman, R. S. 1957. Development of the mapping function at an analytic corner. Pacific J. Math. 7: 1437-1449.

[8] Levin, A. L., E. B. Saff and N. S. Stylianopoulos. 2003. Zero distribution of Bergman orthogonal polynomials for certain planar domains. Const. Approx. 19: 411-435.

[9] Martínez-Finkelshtein, A., K. T.-R. McLaughlin and E. B. Saff. 2006. Szegö orthogonal polynomials with respect to an analytic weight: canonical representation and strong asymptotics. Constr. Approx. 24: 319-363.

[10] Martínez-Finkelshtein, A., K. T.-R. McLaughlin and E. B. Saff. 2006. Asymptotics of orthogonal polynomials with respect to an analytic weight with algebraic singularities on the circle. Int. Math. Res. Notices (doi:10.1155/IMRN/2006/91426).

[11] Maymeskul, V. and E. B. Saff. 2003. Zeros of polynomials orthogonal over regular N-gons. J. Approx. Theory 122: 129-140.

[12] Miña-Díaz, E. 2007. On the asymptotic behavior of Faber polynomials for domains with piecewise analytic boundary. Submitted for publication. arXiv:0706.1806 v1 [math.CA].

[13] Miña-Díaz, E., E. B. Saff and N. S. Stylianopoulos. 2005. Zero distributions for polynomials orthogonal with weights over certain planar regions. CMFT 5: 185-221.

[14] Pommerenke, Ch. 1992. Boundary behavior of conformal maps. New York: Springer-Verlag Berlin Heidelberg.

[15] Saff, E. B., and V. Totik. 1997. Logarithmic potentials with external fields. Berlin: Springer-Verlag. 
[16] Smirnov, V. I., and N. A. Lebedev. 1968. Functions of a complex variable: constructive theory. Cambridge, Massachusetts: The M.I.T. Press.

[17] Simon, B. 2006. Fine structure of the zeros of orthogonal polynomials, I. A tale of two pictures. ETNA 25: 328-368.

[18] Suetin, P. K. 1974. Polynomials orthogonal over a region and Bieberbach polynomials. Proceedings of the Steklov Institute of Mathematics, Amer. Math. Soc., Providence, Rhode Island.

[19] Suetin, P. K. 1998. Series of Faber polynomials. Amsterdam: Gordon and Breach Science Publications.

[20] Szabados, J. 1979. On some problems connected with polynomials orthogonal on the complex unit circle. Act. Math. Scien. Hung. 33: 197-210.

[21] Walsh, J. L. 1969. Interpolation and approximation by rational functions in the complex domain. Amer. Math. Soc. Colloq. Publ. 20, 5th ed., Amer. Math. Soc., Providence, Rhode Island.

Indiana University-Purdue University Fort Wayne, Department of Mathematical Sciences, 2101 E. Coliseum Blvd., Fort Wayne, IN 46805-1499, USA. Email: minae@ipfw.Edu. 